\title{
Design of Effective Zeolite Catalysts for the Complete Hydrogenation of $\mathrm{CO}_{2}$
}

\author{
Bun Chan* and Leo Radom*
}

School of Chemistry, University of Sydney, Sydney, NSW 2006, Australia (E-mail: chan_b@chem.usyd.edu.au; radom@chem.usyd.edu.au)

\section{Supporting Information}

(Tables S1-S3, Total 21 pages) 
Table S1. GAUSSIAN Archive Entries for the B3-LYP/6-31+G(d,p) Optimized Geometries

$\mathbf{H}_{2}$

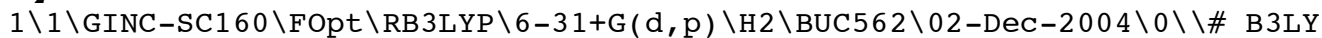
$\mathrm{P} / 6-31+\mathrm{G}(\mathrm{D}, \mathrm{P}) \quad \mathrm{OPT}$ MAXDISK $=65536000 \backslash \backslash \mathrm{H} 2 \backslash \backslash 0,1 \backslash \mathrm{H}, 0 ., 0 ., 0.37139 \backslash \mathrm{H}, 0 ., 0 .,-0$ $.37139 \backslash \backslash$ Version=DEC-AXP-OSF $/ 1-$ G03RevB . 03 \State $=1-$ SGG $\backslash \mathrm{HF}=-1.1785393 \backslash \mathrm{RMS}$ $\mathrm{D}=1.303 \mathrm{e}-10 \backslash \mathrm{RMSF}=5.478 \mathrm{e}-06 \backslash \mathrm{Dipole}=0 ., 0 ., 0 . \backslash \mathrm{PG}=\mathrm{D} * \mathrm{H} \quad[\mathrm{C} *(\mathrm{H} 1 . \mathrm{H} 1)] \backslash \backslash @$

\section{$\mathrm{CO}_{2}$}

$1 \backslash 1 \backslash$ GINC-SC32 $\backslash$ FOpt $\backslash R B 3 L Y P \backslash 6-31+G(d, p) \backslash C 102 \backslash B U C 562 \backslash 03-D e c-2004 \backslash 0 \backslash \backslash \#$ B3L $\mathrm{YP} / 6-31+\mathrm{G}(\mathrm{D}, \mathrm{P})$ OPT MAXDISK $=268435456 \backslash \backslash \mathrm{CO} 2 \backslash \backslash 0,1 \backslash \mathrm{C}, 0 ., 0 ., 0 . \backslash 0,0 ., 0 ., 1.16$ $93585972 \backslash 0,0 ., 0 .,-1.1693585972 \backslash \backslash$ Version=DEC-AXP-OSF/1-G03RevB.03 \State $=1-\mathrm{SGG} \backslash \mathrm{HF}=-188.5903926 \backslash \mathrm{RMSD}=8.870 \mathrm{e}-10 \backslash \mathrm{RMSF}=1.599 \mathrm{e}-05 \backslash \mathrm{Dipole}=0 ., 0 ., 0 . \backslash \mathrm{P}$ $\mathrm{G}=\mathrm{D} * \mathrm{H}[\mathrm{O}(\mathrm{C} 1), \mathrm{C} *(01.01)] \backslash \backslash \mathrm{Q}$

\section{$\mathrm{HCO}_{2} \mathrm{H}$}

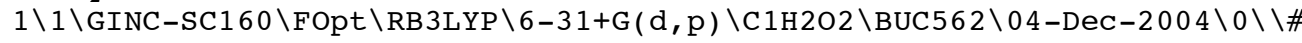
B3LYP $/ 6-31+G(D, P) \quad O P T$ MAXDISK $=268435456 \backslash \backslash \mathrm{HCO} 2 \mathrm{H} \backslash \backslash 0,1 \backslash \mathrm{C},-0.0023942611,0$. $4236059512,0 . \backslash 0,-1.0319846937,-0.4464864955,0 . \backslash 0,1.1645126236,0.115515$ $2786,0 . \backslash \mathrm{H},-0.6611551562,-1.3471024802,0 . \backslash \mathrm{H},-0.3847027163,1.4532365081$, $0 . \backslash \backslash$ Version=DEC-AXP-OSF $/ 1-G 03$ RevB .03 $\backslash$ State $^{\prime}=1-A^{\prime} \backslash \mathrm{HF}=-189.7754948 \backslash \mathrm{RMSD}=3$ $.156 \mathrm{e}-09 \backslash \mathrm{RMSF}=1.486 \mathrm{e}-04 \backslash \mathrm{Dipole}=-0.5896531,-0.1199807,0 . \backslash \mathrm{PG}=\mathrm{CS} \quad[\mathrm{SG}(\mathrm{C} 1 \mathrm{H} 2$ 02 ) $] \backslash \backslash @$

\section{$\mathrm{CH}_{2}(\mathrm{OH})_{2}$}

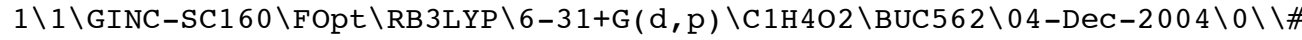
B3LYP $/ 6-31+G(D, P)$ OPT MAXDISK $=268435456 \backslash \backslash \mathrm{CH} 2(\mathrm{OH}) 2 \backslash \backslash 0,1 \backslash \mathrm{C}, 0 ., 0.53240633$ $72,0 . \backslash 0,-1.1723916612,-0.2476784155,-0.0827215773 \backslash 0,1.1723916612,-0.24$ $76784155,0.0827215773 \backslash \mathrm{H},-0.0119891693,1.1597030097,-0.8978585925 \backslash \mathrm{H}, 0.0$ $119891693,1.1597030097,0.8978585925 \backslash \mathrm{H},-1.2461132784,-0.7754946971,0.72$ $51015739 \backslash \mathrm{H}, 1.2461132784,-0.7754946971,-0.7251015739 \backslash \backslash$ Version=DEC $-A X P-O$ $\mathrm{SF} / 1-\mathrm{G} 03 \mathrm{RevB} .03 \backslash$ State $=1-\mathrm{A} \backslash \mathrm{HF}=-190.9676408 \backslash \mathrm{RMSD}=2.835 \mathrm{e}-09 \backslash \mathrm{RMSF}=2.392 \mathrm{e}-0$ $5 \backslash$ Dipole $=0 .,-0.0387797,0 . \backslash P G=C 02 \quad[\mathrm{C} 2(\mathrm{C} 1), \mathrm{X}(\mathrm{H} 4 \mathrm{O} 2)] \backslash \backslash @$

\section{$\mathrm{H}_{2} \mathbf{O}$}

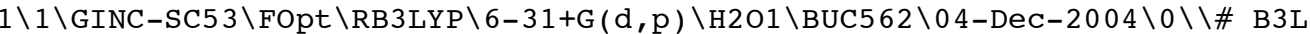
YP $/ 6-31+\mathrm{G}(\mathrm{D}, \mathrm{P})$ OPT MAXDISK $=134217728 \backslash \backslash \mathrm{H} 2 \mathrm{O} \backslash \backslash 0,1 \backslash \mathrm{O}, 0 ., 0 ., 0.1164943011 \backslash \mathrm{H}$, 0 . $0.7694077455,-0.4659772045 \backslash \mathrm{H}, 0,,-0.7694077455,-0.4659772045 \backslash \backslash$ Versio $\mathrm{n}=\mathrm{DEC}-\mathrm{AXP}-\mathrm{OSF} / 1-\mathrm{G} 03 \mathrm{RevB} .03 \backslash \mathrm{State}=1-\mathrm{A} 1 \backslash \mathrm{HF}=-76.4340477 \backslash \mathrm{RMSD}=6.062 \mathrm{e}-09 \backslash \mathrm{RM}$ $\mathrm{SF}=3.817 \mathrm{e}-05 \backslash \mathrm{Dipole}=0 ., 0 .,-0.8636044 \backslash \mathrm{PG}=\mathrm{C} 02 \mathrm{~V} \quad[\mathrm{C} 2(\mathrm{O} 1), \mathrm{SGV}(\mathrm{H} 2)] \backslash \backslash @$

\section{$\mathrm{CH}_{2} \mathrm{O}$}

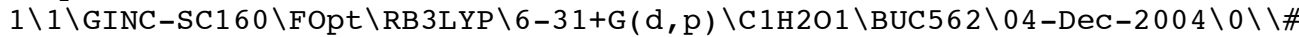
$\mathrm{B} 3 \mathrm{LYP} / 6-31+\mathrm{G}(\mathrm{D}, \mathrm{P})$ OPT MAXDISK $=268435456 \backslash \backslash \mathrm{CH} 2 \mathrm{O} \backslash \backslash 0,1 \backslash \mathrm{C}, 0 ., 0 .,-0.53161522$ $22 \backslash \mathrm{O}, 0 ., 0.0 .6779507378 \backslash \mathrm{H}, 0 ., 0.9411845322,-1.1169572846 \backslash \mathrm{H}, 0 .,-0.941184$ $5322,-1.1169572846 \backslash \backslash$ Version=DEC-AXP-OSF $/ 1-G 03 R e v B .03 \backslash$ State $=1-\mathrm{A} 1 \backslash \mathrm{HF}=-11$ $4.5115243 \backslash \mathrm{RMSD}=3.303 \mathrm{e}-09 \backslash \mathrm{RMSF}=1.267 \mathrm{e}-05 \backslash \mathrm{Dipole}=0 ., 0 .,-0.9896075 \backslash \mathrm{PG}=\mathrm{C} 02$ $\mathrm{V}[\mathrm{C} 2(\mathrm{C} 1 \mathrm{O} 1), \mathrm{SGV}(\mathrm{H} 2)] \backslash \backslash @$

\section{$\mathrm{CH}_{3} \mathrm{OH}$}

$1 \backslash 1 \backslash G I N C-S C 160 \backslash F O p t \backslash R B 3 L Y P \backslash 6-31+G(d, p) \backslash C 1 H 4 O 1 \backslash B U C 562 \backslash 04-D e C-2004 \backslash 0 \backslash \backslash \#$ B3LYP $/ 6-31+G(D, P) \quad O P T$ MAXDISK $=268435456 \backslash \backslash \mathrm{CH} 3 \mathrm{OH} \backslash \backslash 0,1 \backslash \mathrm{C}, 0.050647707,0.66$ $63476588,0 . \backslash \mathrm{O}, 0.0417408203,-0.7583578135,0 . \backslash \mathrm{H},-0.8719707583,-1.0682969$ $078,0 . \backslash \mathrm{H},-0.4326194748,1.0829928137,0.8945466715 \backslash \mathrm{H}, 1.099396904,0.97108$ $7836,0 . \backslash \mathrm{H},-0.4326194748,1.0829928137,-0.8945466715 \backslash \backslash$ Version=DEC-AXP-OS $\mathrm{F} / 1-\mathrm{G} 03$ RevB $.03 \backslash$ State $=1-\mathrm{A}^{\prime} \backslash \mathrm{HF}=-115.7348723 \backslash \mathrm{RMSD}=2.751 \mathrm{e}-09 \backslash \mathrm{RMSF}=6.415 \mathrm{e}-0$ $5 \backslash$ Dipole $=-0.6043467,0.4450933,0 . \backslash P G=C S[S G(C 1 H 2 O 1), x(H 2)] \backslash \backslash @$ 
$1 \backslash 1 \backslash$ GINC-RADOM-BAROSSA0 45 $\backslash$ FOpt $\backslash$ RB 3LYP $\backslash 6-31 \mathrm{G}(\mathrm{d}) \backslash A 11 \mathrm{H} 1104 \mathrm{Si} \backslash$ ELBUNO $\backslash 12-$

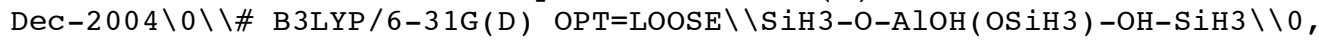
$1 \backslash \mathrm{Al},-0.007655495,0.0094583965,0.344641712 \backslash 0,1.4128279975,-0.482603871$ $7,-0.8631396895 \backslash 0,-1.0731606004,-1.304204499,0.0306424014 \backslash 0,1.13582268$ $57,-0.1271697741,1.6535513885 \backslash 0,-0.576603912,1.5824077875,-0.030336667$ $\backslash \mathrm{Si}, 3.0113267256,-0.6844624266,-0.2005721913 \backslash \mathrm{H}, 3.0966524704,-1.8036912$ $947,0.7525639862 \backslash \mathrm{H}, 3.7783492562,-1.0675771085,-1.419285287 \backslash \mathrm{H}, 3.4761318$ $955,0.627140048,0.2723673995 \backslash \mathrm{Si},-2.4108493001,-2.2305614012,-0.0811175$ $538 \backslash \mathrm{H},-3.3665466325,-1.6982657122,-1.0953255837 \backslash \mathrm{H},-2.0382713184,-3.612$ $0972789,-0.503866182 \backslash \mathrm{H},-3.1334511274,-2.3327736546,1.2193343598 \backslash \mathrm{H}, 1.13$ $25216434,-1.1114948748,-1.5484715978 \backslash \mathrm{Si},-1.1189612876,3.0805301889,-0$. $3877557635 \backslash \mathrm{H},-0.3731749546,3.6623665766,-1.5422126377 \backslash \mathrm{H},-2.5663540903$, $3.0675648948,-0.7475987891 \backslash \mathrm{H},-0.9527870445,4.0045813587,0.772996916 \backslash \mathrm{H}$, $1.1141360413,0.4767616926,2.4056528124 \backslash \backslash$ Version=x86-Linux-G03RevB.02 $\backslash \mathrm{S}$ tate $=1-\mathrm{A} \backslash \mathrm{HF}=-1418.7204941 \backslash \mathrm{RMSD}=3.569 \mathrm{e}-09 \backslash \mathrm{RMSF}=7.420 \mathrm{e}-04 \backslash \mathrm{Dipole}=1.24529$ $05,-0.2818048,-0.3078265 \backslash \mathrm{PG}=\mathrm{C} 01 \quad[\mathrm{X}(\mathrm{Al} 1 \mathrm{H} 1104 \mathrm{Si} 3)] \backslash \backslash \mathrm{C}$ The archive entry for this job was punched.

\section{$1 \cdot \mathrm{CO}_{2}$}

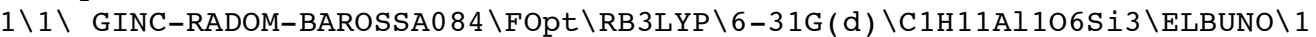

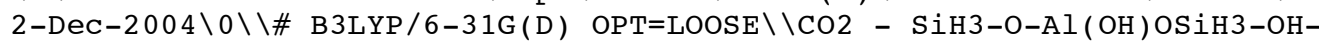
$\mathrm{SiH} 3 \backslash \backslash 0,1 \backslash \mathrm{Al},-0.6646055636,0.0188487573,0.5823848458 \backslash 0,0.0540987537,1$. $6763966699,-0.0530697023 \backslash 0,0.713205357,-1.0170098787,0.3728999781 \backslash 0,-0$ $.9300643342,0.7356695946,2.1518564161 \backslash 0,-2.0692389008,-0.3752154462,-0$ $.3208621603 \backslash \mathrm{Si}, 0.0846161037,3.0134272102,1.0585428441 \backslash \mathrm{H}, 0.9274325272,2$ $.7512288198,2.2383132735 \backslash \mathrm{H}, 0.7435866182,4.0617081515,0.2302365238 \backslash \mathrm{H},-1$ $.2972622411,3.4313832407,1.350355495 \backslash \mathrm{Si}, 1.2551284712,-2.4685333896,0.9$ $292439017 \backslash \mathrm{H}, 0.3762217705,-3.0125786435,2.0060968495 \backslash \mathrm{H}, 1.3047878678,-3$. $4749236413,-0.1683063469 \backslash \mathrm{H}, 2.6310609614,-2.333120729,1.4854446751 \backslash \mathrm{H}, 0$. $7655163147,1.6317604046,-0.7219008797 \backslash 0,2.1578893802,0.9104537322,-1.7$ $703919514 \backslash \mathrm{C}, 2.6826565175,-0.1377570331,-1.6866727187 \backslash 0,3.2415216915,-1$ $.1564826441,-1.6458780282 \backslash \mathrm{Si},-3.1808604329,-0.9658162399,-1.3582386138$ $\backslash \mathrm{H},-3.0963935972,-0.2907149534,-2.6865946567 \backslash \mathrm{H},-3.0021750081,-2.429586$ $7978,-1.5847856782 \backslash \mathrm{H},-4.5596845103,-0.7556754327,-0.830610397 \backslash \mathrm{H},-1.812$ $8310479,0.7044455825,2.540674193 \backslash \backslash$ Version $=x 86-$ Linux-G03RevB. 02 $\backslash$ State $=1$ $-\mathrm{A} \backslash \mathrm{HF}=-1607.3123782 \backslash \mathrm{RMSD}=5.961 \mathrm{e}-09 \backslash \mathrm{RMSF}=1.721 \mathrm{e}-05 \backslash \mathrm{Dipole}=0.4776967,1.2$ $084312,-0.1752802 \backslash \mathrm{PG}=\mathrm{C} 01 \quad[\mathrm{X}(\mathrm{C} 1 \mathrm{H} 11 \mathrm{Al} 1 \mathrm{O} 6 \mathrm{Si3})] \backslash \backslash @$

\section{$\operatorname{TS}\left(1, \mathrm{CO}_{2}\right)$}

$1 \backslash 1 \backslash$ GINC-RADOM-BAROSSA082 $\backslash F T S \backslash R B 3 L Y P \backslash 6-31 \mathrm{G}(\mathrm{d}) \backslash \mathrm{C} 1 \mathrm{H} 13 \mathrm{~A} 1106 \mathrm{Si} \backslash \mathrm{ELBUNO} \backslash 13$

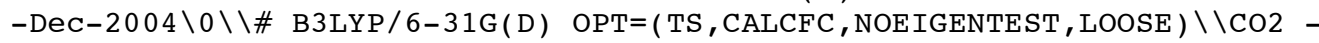
$>\mathrm{HCO} 2 \mathrm{H}$ TS $(\mathrm{SiH} 3-\mathrm{O}-\mathrm{AlOH}(\mathrm{OSiH} 3)-\mathrm{OH}-\mathrm{SiH} 3) \backslash \backslash 0,1 \backslash \mathrm{Al},-0.8146338179,-0.19822$ $30466,0.4883591995 \backslash 0,0.1792113142,1.3825898429,0.458414133 \backslash 0,0.5253472$ $272,-1.4047987944,0.2728251328 \backslash 0,-1.3230363302,-0.2014848264,2.1381267$ $402 \backslash 0,-1.9393305973,-0.2272028502,-0.7917928109 \backslash \mathrm{Si}, 0.2066184192,2.5028$ $483576,1.7690498728 \backslash \mathrm{H}, 0.8366005579,1.8856545224,2.9535949852 \backslash \mathrm{H}, 1.02543$ $33891,3.6322663489,1.2715073359 \backslash \mathrm{H},-1.1805732234,2.9342582809,2.0324345$ $348 \backslash \mathrm{Si}, 0.7725900127,-2.8924359254,1.0496402504 \backslash \mathrm{H}, 1.7524760284,-3.64065$ $46812,0.226433837 \backslash \mathrm{H}, 1.3098015878,-2.6874904783,2.4157927623 \backslash \mathrm{H},-0.51918$ $08972,-3.6169718944,1.1090726321 \backslash \mathrm{H}, 0.9290899619,1.4810755224,-0.242755$ $191 \backslash 0,2.0741750636,1.5645279723,-1.2287528914 \backslash \mathrm{C}, 2.7543461519,0.5947078$ $699,-1.5520894684 \backslash 0,3.7073294002,0.2392172163,-2.1680035352 \backslash \mathrm{H}, 2.174349$ $2292,-0.5600365996,-0.9626764358 \backslash \mathrm{H}, 1.4844789673,-0.9792445636,-0.43628$ $7276 \backslash \mathrm{Si},-2.9414513506,-0.2664300764,-2.0840671229 \backslash \mathrm{H},-2.8322870456,0.98$ $7132289,-2.8816083244 \backslash \mathrm{H},-2.6175018243,-1.4143054201,-2.9772006651 \backslash \mathrm{H},-4$ $.3579235667,-0.4116694558,-1.6433894231 \backslash \mathrm{H},-2.2587682039,-0.239902948,2$ $.3676922926 \backslash \backslash$ Version=x86-Linux-G0 3RevB. 02 $\backslash$ State $=1-A \backslash H F=-1608.4347705 \backslash R$ $\mathrm{MSD}=7.255 \mathrm{e}-09 \backslash \mathrm{RMSF}=8.005 \mathrm{e}-06 \backslash \mathrm{Dipole}=-0.846567,-0.0632832,1.3981394 \backslash \mathrm{PG}=$ $\mathrm{C} 01[\mathrm{X}(\mathrm{C} 1 \mathrm{H} 13 \mathrm{Al} 106 \mathrm{Si3})] \backslash \backslash @$ 


\section{$1 \cdot \mathrm{HCO}_{2} \mathrm{H}$}

$1 \backslash 1 \backslash$ GINC-RADOM-BAROSSA0 80 \FOpt \RB3LYP \6-31G(d) \C1H13Al106Si3

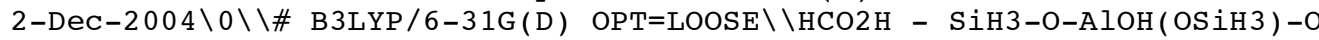
$\mathrm{H}-\mathrm{SiH} 3 \backslash \backslash 0,1 \backslash \mathrm{Al},-0.9890604942,0.0024144744,-0.1600936486 \backslash 0,0.1197462981$ $,-1.1772140686,-0.8766801719 \backslash 0,0.1680625653,1.5232242782,-0.1028988152$ $\backslash 0,-2.1208570754,0.7227850123,-1.257740633 \backslash 0,-1.5026802055,-0.37835876$ $82,1.4265779422 \backslash \mathrm{Si},-0.0933702076,-2.4342458837,-1.9410934002 \backslash \mathrm{H}, 0.00043$ $62843,-1.9731559341,-3.3525786949 \backslash \mathrm{H}, 0.9847479534,-3.4342806743,-1.7059$ $363947 \backslash \mathrm{H},-1.4125217533,-3.0924577411,-1.7378893929 \backslash \mathrm{Si},-0.2659482925,2$. $9877029395,-0.9214772798 \backslash \mathrm{H},-1.5011352218,3.4960891944,-0.2987857987 \backslash \mathrm{H}$, $0.8749836843,3.8906687078,-0.6285695076 \backslash \mathrm{H},-0.377073306,2.7758807839,-2$ $.3768279577 \backslash \mathrm{H}, 1.6871747466,-0.6311979852,-0.5504816534 \backslash 0,2.3620474541$, $0.0602807444,-0.2813733427 \backslash C, 3.395880111,-0.4412715206,0.4389812008 \backslash 0$, $4.2387039054,0.2526002665,0.9333396892 \backslash \mathrm{H}, 3.3761300777,-1.5433959192,0$. $5035632015 \backslash \mathrm{H}, 1.1335531711,1.2985814191,-0.0536596765 \backslash \mathrm{Si},-1.7676838459$, $-0.8389340031,2.9708402049 \backslash \mathrm{H},-0.7788352416,-1.8658243641,3.4103461663 \backslash$ $\mathrm{H},-1.6590375697,0.3218623356,3.9001191724 \backslash \mathrm{H},-3.1307204133,-1.422390280$ $8,3.1233105174 \backslash \mathrm{H},-3.0573473445,0.7659989598,-1.0308504516 \backslash \backslash$ Version $=\mathrm{x} 86$ - Linux-G03RevB. 02 $\backslash$ State $=1-A \backslash H F=-1608.4957809 \backslash \mathrm{RMSD}=6.375 \mathrm{e}-09 \backslash \mathrm{RMSF}=4.935$ e-05\Dipole $=-0.5911522,0.254923,-0.6102854 \backslash P G=C 01 \quad[X(C 1 H 13 A l 106 \mathrm{Si})] \backslash \backslash$ a

\section{TS $\left(1, \mathrm{HCO}_{2} \mathrm{H}\right)$}

$1 \backslash 1 \backslash$ GINC-RADOM-BAROSSA $18 \backslash \mathrm{FTS} \backslash \mathrm{RB} 3 \mathrm{LYP} \backslash 6-31 \mathrm{G}(\mathrm{d}) \backslash \mathrm{C} 1 \mathrm{H} 15 \mathrm{~A} 1106 \mathrm{Si} \backslash \mathrm{ELBUNO} \backslash 14$

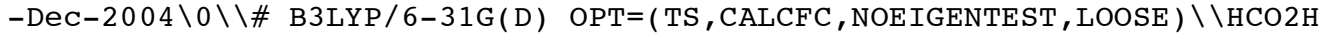
$\rightarrow \mathrm{CH} 2(\mathrm{OH}) 2$ TS (SiH3-O-AlOH(OSiH3)-OH-SiH3) $\backslash \backslash 0,1 \backslash \mathrm{Al},-0.861350314,0.26$ $59427938,0.0526167584 \backslash 0,-0.0155305186,-0.5469349293,1.4177335941 \backslash 0,-0$. $5085406184,-0.8551420264,-1.3442886147 \backslash 0,-2.5600489088,0.3020841393,0$. $3654508793 \backslash 0,0.0352652644,1.7154423991,-0.3148197406 \backslash \mathrm{Si},-0.5489879578$, $-0.8833869652,2.9629632908 \backslash \mathrm{H},-1.0752549593,0.335854337,3.6300293438 \backslash \mathrm{H}$, $-1.5980736104,-1.9354241743,2.9656900734 \backslash \mathrm{H}, 0.624535032,-1.3812614763,3$ $.7335436417 \backslash \mathrm{Si},-1.5529450088,-1.8387825171,-2.2132317514 \backslash \mathrm{H},-2.25547067$ $34,-2.7987663038,-1.3257800042 \backslash \mathrm{H},-2.5518465702,-1.0300679503,-2.957991$ $3338 \backslash \mathrm{H},-0.7185168522,-2.5945630978,-3.1858585496 \backslash \mathrm{H}, 1.3349773345,-0.938$ $1942706,1.0108485726 \backslash 0,2.2759672373,-1.1929607233,0.6094862494 \backslash \mathrm{C}, 2.606$ $5053923,-0.4254270347,-0.3836909392 \backslash 0,3.8264896364,-0.5896786342,-0.88$ $68722881 \backslash \mathrm{H}, 1.7243414697,-0.8651105426,-1.584935696 \backslash \mathrm{H}, 0.8251906443,-0.9$ $071112793,-1.5865284327 \backslash \mathrm{H}, 2.2395123276,0.599569797,-0.4434057819 \backslash \mathrm{H}, 4.1$ $481637351,-1.4726217555,-0.6229088895 \backslash \mathrm{Si}, 0.0545865564,3.3477886236,-0$. $4204805555 \backslash \mathrm{H}, 1.213193888,3.7901326703,-1.2494487656 \backslash \mathrm{H}, 0.1877330692,3.9$ $954225572,0.9176097771 \backslash \mathrm{H},-1.1874964115,3.8855605867,-1.0528045745 \backslash \mathrm{H},-3$ $.1184336893,0.9907370119,-0.012966014 \backslash \backslash$ Version=x86-Linux-G03RevB.02\St ate $=1-\mathrm{A} \backslash \mathrm{HF}=-1609.6186864 \backslash \mathrm{RMSD}=4.869 \mathrm{e}-09 \backslash \mathrm{RMSF}=1.365 \mathrm{e}-05 \backslash \mathrm{Dipole}=1.154451$ $3,-0.623735,-0.3787196 \backslash P G=C 01 \quad[X(C 1 H 15 A 1106 \mathrm{Si3})] \backslash \backslash @$

\section{$1 \cdot \mathrm{CH}_{2}(\mathrm{OH})_{2}$}

$1 \backslash 1 \backslash$ GINC-RADOM-BAROSSA1 17 \FOpt \RB3LYP \6-31G(d) \C1H15Al106Si3 \ELBUNO \1

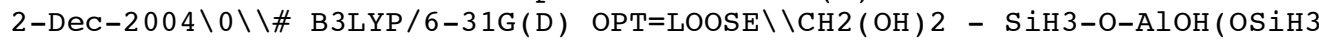
) $-\mathrm{OH}-\mathrm{SiH} 3 \backslash \backslash 0,1 \backslash \mathrm{Al},-0.9097493913,0.0462772005,-0.4601360443 \backslash 0,0.4907342$ $979,-0.9712382975,-0.7990659749 \backslash 0,0.014537713,1.6305506181,0.055101330$ $2 \backslash 0,-1.7122978578,0.808293072,-1.7978918842 \backslash 0,-1.8893581115,-0.5402994$ $17,0.8207153186 \backslash \mathrm{Si}, 0.7205468858,-2.5442605888,-1.2632307848 \backslash \mathrm{H},-0.49524$ $7628,-3.0869985333,-1.9335295894 \backslash \mathrm{H}, 1.8596520976,-2.6181515741,-2.21830$ $44509 \backslash \mathrm{H}, 1.0361882433,-3.4100383079,-0.0944314036 \backslash \mathrm{Si},-0.3877767246,3.13$ $54905098,-0.6865483073 \backslash \mathrm{H},-1.8007737295,3.4302760233,-0.3871033645 \backslash \mathrm{H}, 0$. $4896079554,4.108868228,0.0166503962 \backslash \mathrm{H},-0.0685798166,3.1505260517,-2.12$ $72283916 \backslash \mathrm{H}, 1.9167563748,-0.0637744189,-0.320718667 \backslash 0,2.409797964,0.673$ $3570483,0.1313572835 \backslash C, 3.0572819383,0.1167487742,1.2725206928 \backslash 0,3.9492$ $777012,-0.8945710761,0.911775367 \backslash \mathrm{H}, 3.5471378818,0.963153946,1.76942980$ $17 \backslash \mathrm{H}, 0.9968386185,1.4629199256,0.2425045819 \backslash \mathrm{H}, 2.3376789254,-0.35225881$ $18,1.9518426154 \backslash \mathrm{H}, 4.6290060485,-0.4997800006,0.3408877676 \backslash \mathrm{Si},-2.831405$ $757,-1.0835883667,2.0339892245 \backslash \mathrm{H},-2.5913930185,-2.5312731009,2.3005335$ $32 \backslash \mathrm{H},-2.5656877176,-0.3404767191,3.3002377921 \backslash \mathrm{H},-4.279108886,-0.920477$ $2971,1.7101451833 \backslash \mathrm{H},-2.6496602048,0.6496709945,-1.95914476 \backslash \backslash$ Version $=x 8$ 6-Linux-G03RevB.02 \State $=1-A \backslash H F=-1609.6866104 \backslash \mathrm{RMSD}=4.764 \mathrm{e}-09 \backslash \mathrm{RMSF}=2.18$ $7 e-04 \backslash \mathrm{Dipole}=0.7015397,1.045299,0.0206286 \backslash \mathrm{PG}=\mathrm{C} 01 \quad[\mathrm{X}(\mathrm{C} 1 \mathrm{H} 15 \mathrm{Al} 106 \mathrm{Si} 3)] \backslash \backslash @$ 


\section{$1 \cdot \mathrm{CH}_{2} \mathrm{O}$}

$1 \backslash 1 \backslash$ GINC-SC2 7 $\backslash F O p t \backslash R B 3 L Y P \backslash 6-31+G(d, p) \backslash C 1 H 13 A 1105 S i 3 \backslash B U C 562 \backslash 01-M a r-2005$ $\backslash 0 \backslash \backslash \#$ B3LYP/6-31+G(D,P) OPT MAXDISK=268435456\\CH2O SiH3-O-AlOH(OSiH3)

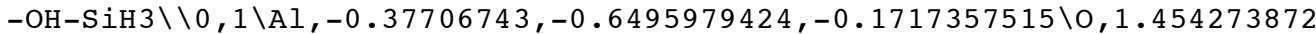
$4,-0.1194787623,-0.2177485482 \backslash 0,-1.1595440118,0.7390786706,-0.86924932$ $98 \backslash 0,-0.1195525259,-2.0440408625,-1.1797986681 \backslash 0,-0.7878543646,-0.8941$ $111603,1.4799060413 \backslash \mathrm{Si}, 2.698498428,-1.0979986561,-0.9023551792 \backslash \mathrm{H}, 2.721$ $1462803,-2.3909755686,-0.1967476253 \backslash \mathrm{H}, 2.4975057067,-1.2357066605,-2.35$ $57799333 \backslash \mathrm{H}, 3.9370667974,-0.3303536777,-0.624362014 \backslash \mathrm{Si},-2.1139624505,1$. $38464297,-2.0368294855 \backslash \mathrm{H},-3.5421166501,1.0071798072,-1.8540093027 \backslash \mathrm{H},-2$ $.0182982368,2.8726232049,-1.9590811737 \backslash \mathrm{H},-1.6916261715,0.9708973751,-3$ $.4027483987 \backslash \mathrm{H}, 1.661631208,0.8238823694,0.0348363551 \backslash 0,1.7575430958,2.4$ $190283914,0.4864399496 \backslash \mathrm{C}, 0.7279885307,3.0764579715,0.4987570385 \backslash \mathrm{H}, 0.75$ $30518907,4.1327987773,0.8141310261 \backslash \mathrm{H},-0.2346414405,2.6347316992,0.1950$ $527227 \backslash \mathrm{Si},-1.1772032819,-1.1841650035,3.0360780664 \backslash \mathrm{H}, 0.0415740552,-1.4$ $769426832,3.8429356883 \backslash \mathrm{H},-1.852914139,-0.0034619498,3.6454427135 \backslash \mathrm{H},-2$. $0961661531,-2.3510061704,3.1518281037 \backslash \mathrm{H},-0.5038540067,-2.9061616616,-1$ $.0043788045 \backslash \backslash$ Version=DEC-AXP-OSF / 1-G03RevB. 03 $\backslash$ State $=1-A \backslash H F=-1533.29980$ $5 \backslash \mathrm{RMSD}=3.909 \mathrm{e}-09 \backslash \mathrm{RMSF}=8.826 \mathrm{e}-05 \backslash \mathrm{Dipole}=0.8172675,0.8586564,-0.0188893 \backslash$ $P G=C 01 \quad[X(C 1 H 13 A 1105 S i 3)] \backslash \backslash @$

\section{$\operatorname{TS}\left(1, \mathrm{CH}_{2} \mathrm{O}\right)$}

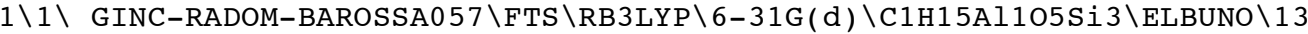

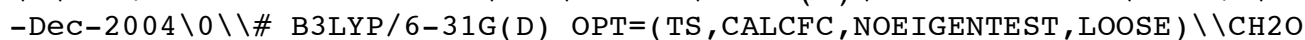
$\rightarrow \mathrm{CH} 3 \mathrm{OH}$ TS ( $\mathrm{SiH} 3-\mathrm{O}-\mathrm{AlOH}(\mathrm{OSiH} 3)-\mathrm{OH}-\mathrm{SiH} 3) \backslash \backslash 0,1 \backslash \mathrm{Al}, 0.512862112,0.3549807$ $623,0.0689156494 \backslash 0,-0.6627956229,-0.2979715441,1.2925619375 \backslash 0,-0.57921$ $83174,1.0528605568,-1.1909595438 \backslash 0,1.4446277383,1.5643739773,0.8781170$ $151 \backslash 0,1.268908794,-1.0236669455,-0.6837389713 \backslash \mathrm{Si},-0.6136751939,-0.1336$ $568843,2.960552098 \backslash \mathrm{H}, 0.7019498432,-0.5663360764,3.4945546301 \backslash \mathrm{H},-0.8923$ $276601,1.2616874412,3.3816249709 \backslash \mathrm{H},-1.6752091657,-1.0228320217,3.50781$ $3613 \backslash \mathrm{Si},-0.9164824826,2.6450652894,-1.5622219172 \backslash \mathrm{H}, 0.2812980962,3.3422$ $363047,-2.1012346331 \backslash \mathrm{H},-1.9755209571,2.6373278566,-2.6086888722 \backslash \mathrm{H},-1.4$ $193875858,3.3891926605,-0.3782946135 \backslash \mathrm{H},-1.7097203905,-0.9077350864,0.6$ $905837045 \backslash 0,-2.4883473841,-1.3601097226,0.0559024768 \backslash \mathrm{C},-1.9877399619,-$ $1.8407378669,-1.0127907189 \backslash \mathrm{H},-2.6719560155,-2.361757577,-1.684197082 \backslash \mathrm{H}$ $,-1.8068963699,-0.6881324079,-2.1323630191 \backslash \mathrm{H},-1.3612489772,-0.00832113$ $6,-1.8865850981 \backslash \mathrm{H},-0.9212648883,-2.076705958,-1.0627561869 \backslash \mathrm{Si}, 2.623361$ $0553,-1.8892625958,-0.9817444264 \backslash \mathrm{H}, 2.9770373331,-2.7865770306,0.157649$ $6931 \backslash \mathrm{H}, 2.4231687074,-2.7536508011,-2.1816611989 \backslash \mathrm{H}, 3.8061631806,-1.0159$ $659609,-1.2452088845 \backslash \mathrm{H}, 2.3328981972,1.7933151777,0.5823399695 \backslash \backslash$ Version $=x 86-$ Linux-G03RevB. 02 $\backslash$ State $=1-A \backslash H F=-1534.383054 \backslash R M S D=6.152 e-09 \backslash R M S F=1$. $904 \mathrm{e}-05 \backslash \mathrm{Dipole}=-0.7457506,-0.7411459,-0.633953 \backslash \mathrm{PG}=\mathrm{C} 01]$ [X(C1H15A]105Si3 ) $] \backslash \backslash$ @

\section{$1 \cdot \mathrm{CH}_{3} \mathrm{OH}$}

$1 \backslash 1 \backslash$ GINC-RADOM-BAROSSA050 \FOpt \RB3LYP \6-31G(d) \C1H15Al105Si3 \ELBUNO \1

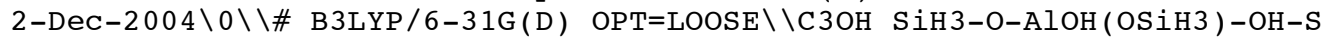
$\mathrm{iH} 3 \backslash \backslash 0,1 \backslash \mathrm{Al},-0.7712746788,0.1033368115,-0.2295471625 \backslash 0,0.2823617838,-1$ $.1104455532,-0.9628703239 \backslash 0,0.5277679705,1.4558234534,0.0765674903 \backslash 0,-$ $1.7785422054,1.0080381667,-1.3133608957 \backslash 0,-1.4589977614,-0.3689313009$, $1.274359411 \backslash \mathrm{Si}, 0.04822458,-2.3381652339,-2.046119742 \backslash \mathrm{H}, 0.2908011216,-1$ $.8880718131,-3.4449659254 \backslash \mathrm{H}, 1.0096527263,-3.4372823813,-1.7474704442 \backslash \mathrm{H}$ $,-1.3339070194,-2.8886236467,-1.9630232279 \backslash \mathrm{Si}, 0.3173284316,3.048510221$ $6,-0.53329877 \backslash \mathrm{H},-0.90203756,3.6101778129,0.0791132529 \backslash \mathrm{H}, 1.5152596771,3$ $.7855682689,-0.053798111 \backslash \mathrm{H}, 0.2823882717,3.068185416,-2.0094683841 \backslash \mathrm{H}, 1$. $9835254683,-0.6261844239,-0.553928834 \backslash 0,2.6170289045,0.0206495198,-0.1$ $554556408 \backslash \mathrm{C}, 3.226327154,-0.5680422061,1.0000311083 \backslash \mathrm{H}, 3.8499872057,0.20$ $01438458,1.4620860797 \backslash \mathrm{H}, 2.4794677965,-0.9122414577,1.7264009318 \backslash \mathrm{H}, 1.47$ $26552072,1.0552345371,0.0700659477 \backslash \mathrm{H}, 3.8619866426,-1.411260085,0.70771$ $21093 \backslash \mathrm{Si},-1.6231800248,-0.9974646049,2.7694586862 \backslash \mathrm{H},-0.3041832188,-1.3$ $908457899,3.3545226643 \backslash \mathrm{H},-2.2421104971,-0.0092460146,3.6991132268 \backslash \mathrm{H},-2$ $.4871012804,-2.21262274,2.7574258023 \backslash \mathrm{H},-2.7179479933,1.100543512,-1.11$ $43413905 \backslash \backslash$ Version $=x 86-$ Linux $-G 03$ RevB. $02 \backslash$ State $=1-A \backslash H F=-1534.4682366 \backslash$ RMSD $=4.433 e-09 \backslash \mathrm{RMSF}=3.977 \mathrm{e}-05 \backslash \mathrm{Dipole}=0.8553457,0.4907027,0.2801384 \backslash \mathrm{PG}=\mathrm{C} 01$ $[\mathrm{X}(\mathrm{C} 1 \mathrm{H} 15 \mathrm{Al105Si3)}] \backslash \backslash @$ 


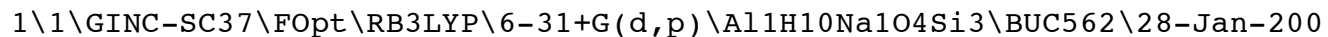

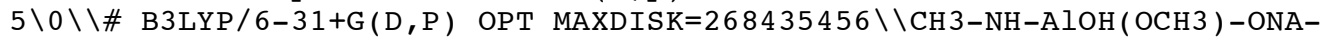
$\mathrm{CH} 3 \backslash \backslash 0,1 \backslash \mathrm{Si},-0.0522761249,3.2165245691,-0.593333918 \backslash 0,-0.0221006093,1$. $6725919957,-0.0836210399 \backslash \mathrm{Al}, 0.0005690847,0.0648639947,0.5595302969 \backslash 0,-$ $1.3253233523,-0.9338255255,-0.1784306432 \backslash \mathrm{Si},-2.9693232738,-0.957538118$ $2,-0.0027997066 \backslash \mathrm{Na}, 0.0341978855,-2.2039884103,-1.3161392098 \backslash 0,0.000474$ $3888,-0.0721428673,2.2924174383 \backslash 0,1.3542348669,-0.8979948593,-0.174141$ $3303 \backslash \mathrm{H},-3.5718131241,0.3907644932,-0.1692121074 \backslash \mathrm{H},-3.5176503635,-1.851$ $3234665,-1.0688897842 \backslash \mathrm{H},-3.3862680194,-1.5104791858,1.3120782104 \backslash \mathrm{H}, 1.3$ $178908589,3.7049757319,-0.9230960444 \backslash \mathrm{H},-0.8864889348,3.3608601104,-1.8$ $221491611 \backslash \mathrm{H},-0.6178888486,4.1256924142,0.4451748747 \backslash \mathrm{Si}, 2.9983308755,-0$ $.8953873273,-0.0005424769 \backslash \mathrm{H},-0.0181266291,0.705329101,2.8558967522 \backslash \mathrm{H}, 3$ $.4242612504,-1.3209400357,1.3576437809 \backslash \mathrm{H}, 3.591577251,0.4350170802,-0.2$ $95910162 \backslash \mathrm{H}, 3.548408689,-1.8786733408,-0.9842288887 \backslash \backslash$ Version=DEC-AXP-OS $\mathrm{F} / 1-\mathrm{G} 03$ RevB $.03 \backslash$ State $=1-\mathrm{A} \backslash \mathrm{HF}=-1580.5556216 \backslash \mathrm{RMSD}=8.189 \mathrm{e}-09 \backslash \mathrm{RMSF}=3.825 \mathrm{e}-0$ $5 \backslash$ Dipole $=0.0367377,-2.2880503,-1.8219233 \backslash P G=C 01 \quad[\mathrm{X}(\mathrm{Al} 1 \mathrm{H} 10 \mathrm{Na1O4Si3})] \backslash \backslash @$

\section{$2 \cdot \mathrm{CO}_{2}$}

$1 \backslash 1 \backslash$ GINC-SC2 2 FOpt $\backslash R B 3 L Y P \backslash 6-31+G(d, p) \backslash C 1 H 10 A 11 N a 106$ Si3 $\backslash B U C 562 \backslash 28-J a n-20$ $05 \backslash 0 \backslash \backslash \#$ B3LYP/6-31+G(D,P) OPT MAXDISK=268435456\\CO2 - SiH3-O-AlOH (OSi

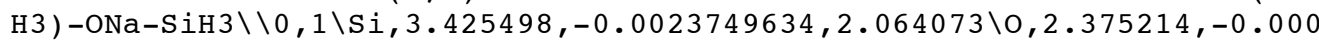
$3409746,0.8242 \backslash \mathrm{Al}, 1.35138,0.0011840144,-0.575164 \backslash 0,0.1382990143,-1.341$ $9869985,-0.466045 \backslash \mathrm{Si}, 0.2456840318,-2.9750759974,-0.691385 \backslash \mathrm{Na},-1.556887$ $,-0.0005700166,-0.063596 \backslash 0,-3.843896,-0.0024020411,0.455161 \backslash \mathrm{C},-4.99131$ $2,-0.0012990533,0.708194 \backslash 0,-6.124815,-0.0002640654,0.958107 \backslash 0,2.163391$ $, 0.0043220231,-2.113771 \backslash 0,0.1370489857,1.3430190015,-0.462984 \backslash \mathrm{H},-0.903$ $4299614,-3.6133190097,0.020142 \backslash \mathrm{H}, 0.1397540357,-3.3446239985,-2.128565 \backslash$ $\mathrm{H}, 1.5072650378,-3.5396339839,-0.145314 \backslash \mathrm{H}, 4.839818,-0.0034479483,1.5892$ $01 \backslash \mathrm{H}, 3.2432299872,1.1995190347,2.929733 \backslash \mathrm{H}, 3.2408000129,-1.2053019654,2$ $.9278 \backslash \mathrm{Si}, 0.2441809682,2.9762380026,-0.68797 \backslash \mathrm{H}, 3.122539,0.0026550334,-2$ $.165952 \backslash \mathrm{H}, 0.1407959643,3.3460800015,-2.125232 \backslash \mathrm{H},-0.9065470386,3.613740$ $9903,0.021619 \backslash \mathrm{H}, 1.5044559622,3.5412010161,-0.139306 \backslash \backslash$ Version=DEC-AXP-O $\mathrm{SF} / 1-\mathrm{G} 03 \mathrm{RevB} .03 \backslash \mathrm{State}=1-\mathrm{A} \backslash \mathrm{HF}=-1769.1570316 \backslash \mathrm{RMSD}=3.043 \mathrm{e}-09 \backslash \mathrm{RMSF}=9.074 \mathrm{e}-$ $06 \backslash$ Dipole $=-3.4369779,-0.0034503,0.5885474 \backslash \mathrm{PG}=\mathrm{C} 01 \quad[\mathrm{X}(\mathrm{C} 1 \mathrm{H} 10 \mathrm{Al} 1 \mathrm{Na} 106 \mathrm{Si3})]$ $\backslash \backslash$ @

\section{$\operatorname{TS}\left(2, \mathrm{CO}_{2}\right)$}

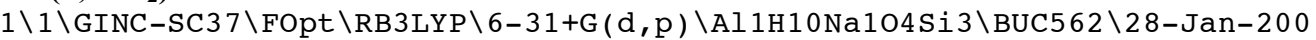

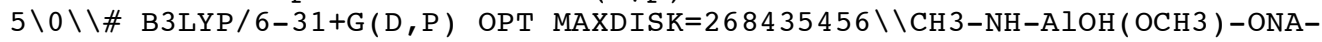
$\mathrm{CH} 3 \backslash \backslash 0,1 \backslash \mathrm{Si},-0.0522761249,3.2165245691,-0.593333918 \backslash 0,-0.0221006093,1$. $6725919957,-0.0836210399 \backslash \mathrm{Al}, 0.0005690847,0.0648639947,0.5595302969 \backslash 0,-$ $1.3253233523,-0.9338255255,-0.1784306432 \backslash \mathrm{Si},-2.9693232738,-0.957538118$ $2,-0.0027997066 \backslash \mathrm{Na}, 0.0341978855,-2.2039884103,-1.3161392098 \backslash 0,0.000474$ $3888,-0.0721428673,2.2924174383 \backslash 0,1.3542348669,-0.8979948593,-0.174141$ $3303 \backslash \mathrm{H},-3.5718131241,0.3907644932,-0.1692121074 \backslash \mathrm{H},-3.5176503635,-1.851$ $3234665,-1.0688897842 \backslash \mathrm{H},-3.3862680194,-1.5104791858,1.3120782104 \backslash \mathrm{H}, 1.3$ $178908589,3.7049757319,-0.9230960444 \backslash \mathrm{H},-0.8864889348,3.3608601104,-1.8$ $221491611 \backslash \mathrm{H},-0.6178888486,4.1256924142,0.4451748747 \backslash \mathrm{Si}, 2.9983308755,-0$ $.8953873273,-0.0005424769 \backslash \mathrm{H},-0.0181266291,0.705329101,2.8558967522 \backslash \mathrm{H}, 3$ $.4242612504,-1.3209400357,1.3576437809 \backslash \mathrm{H}, 3.591577251,0.4350170802,-0.2$ $95910162 \backslash \mathrm{H}, 3.548408689,-1.8786733408,-0.9842288887 \backslash \backslash$ Version=DEC-AXP-OS $\mathrm{F} / 1-\mathrm{G} 03$ RevB.03 $\backslash$ State $=1-\mathrm{A} \backslash \mathrm{HF}=-1580.5556216 \backslash \mathrm{RMSD}=8.189 \mathrm{e}-09 \backslash \mathrm{RMSF}=3.825 \mathrm{e}-0$ $5 \backslash$ Dipole $=0.0367377,-2.2880503,-1.8219233 \backslash P G=C 01 \quad[X(A l 1 H 10 N a 104 S i 3)] \backslash \backslash @$ 


\section{$2 \cdot \mathrm{HCO}_{2} \mathrm{H}$}

$1 \backslash 1 \backslash G I N C-S C 16 \backslash F O p t \backslash R B 3 L Y P \backslash 6-31+G(d, p) \backslash C 1 H 12 A 11 N a 106 S i 3 \backslash B U C 562 \backslash 28-J a n-2$ $005 \backslash 0 \backslash \backslash \#$ B3LYP/6-31+G(D,P) OPT=LOOSE MAXDISK=268435456\\HCO2H - SiH3-O

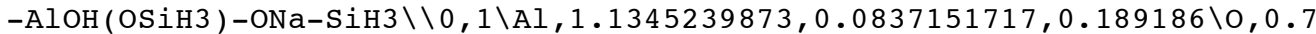
$912691876,-1.2390738802,-0.989866 \backslash 0,0.0055070107,-0.0706879992,1.52948$ $6 \backslash 0,2.7633049704,0.1957744183,0.773087 \backslash 0,0.5635247838,1.4280920853,-0$. $871357 \backslash \mathrm{Si}, 1.1740774298,-2.8392548223,-1.11841 \backslash \mathrm{H}, 0.845092545,-3.6008508$ $721,0.115773 \backslash \mathrm{H}, 0.3773945164,-3.4116909429,-2.244911 \backslash \mathrm{H}, 2.6146454604,-3$. $0417906042,-1.431058 \backslash \mathrm{Si}, 0.0427110253,-0.1674719935,3.173357 \backslash \mathrm{H},-1.35781$ $79499,-0.3310152055,3.662087 \backslash \mathrm{H}, 0.8312882022,-1.3355798742,3.652737 \backslash \mathrm{H}, 0$ $.6014558392,1.062672091,3.79853 \backslash \mathrm{Na},-0.6161410059,0.0392889067,-2.17806$ $1 \backslash 0,-2.6573299766,-0.1542814022,-1.197098 \backslash \mathrm{C},-2.8054529689,-0.205701424$ $6,0.01806 \backslash 0,-3.9988049536,-0.3068486053,0.59736 \backslash \mathrm{H},-1.9804329736,-0.174$ $3312998,0.745693 \backslash \mathrm{H},-4.6831379501,-0.3298467089,-0.096667 \backslash \mathrm{Si}, 0.66214853$ $45,3.0753021002,-0.851026 \backslash \mathrm{H}, 3.5393769566,0.2866365357,0.215991 \backslash \mathrm{H}, 2.049$ $0294623,3.5521853101,-1.101639 \backslash \mathrm{H},-0.2069285448,3.5993519687,-1.947134 \backslash$ $\mathrm{H}, 0.1865984469,3.6541280282,0.433687 \backslash \backslash$ Version=DEC-AXP-OSF / 1-G03RevB. 03 $\backslash$ State $=1-\mathrm{A} \backslash \mathrm{HF}=-1770.3568516 \backslash \mathrm{RMSD}=8.515 \mathrm{e}-09 \backslash \mathrm{RMSF}=1.964 \mathrm{e}-05 \backslash \mathrm{Dipole}=-1.83$ $31221,-0.0356638,-2.4448218 \backslash \mathrm{PG}=\mathrm{C} 01$ [X(C1H12A11Na106Si3)] \@

\section{$\operatorname{TS}\left(2, \mathrm{HCO}_{2} \mathrm{H}\right)$}

$1 \backslash 1 \backslash G I N C-S C 123 \backslash F T S \backslash R B 3 L Y P \backslash 6-31+G(d, p) \backslash C 1 H 14 A 11 N a 106 S i 3 \backslash B U C 562 \backslash 24-J a n-2$ $005 \backslash 0 \backslash \backslash \#$ B3LYP $/ 6-31+G(D, P) \quad O P T=(T S, C A L C F C, N O E I G E N T E S T)$ MAXDISK=6710886 $40 \backslash \backslash \mathrm{HCO} 2 \mathrm{H} \rightarrow \mathrm{CH} 2(\mathrm{OH}) 2 \mathrm{TS}(\mathrm{SiH} 3-\mathrm{O}-\mathrm{AlOH}(\mathrm{OSiH} 3)-\mathrm{ONa}-\mathrm{SiH} 3) \backslash \backslash 0,1 \backslash \mathrm{Al}, 1.20390$ $6364,0.0273348559,0.0801127773 \backslash 0,0.5062462875,-0.4448316548,1.63808416$ $56 \backslash 0,0.1840068386,1.3996561149,-0.5887537226 \backslash 0,2.787427599,0.709594220$ $5,-0.0029178235 \backslash 0,0.796969339,-1.4250919927,-0.8490460314 \backslash \mathrm{Si}, 0.4359223$ $448,0.0802447654,3.2041891463 \backslash \mathrm{H},-0.0104467139,1.4958640606,3.293296581$ $9 \backslash \mathrm{H},-0.5428516346,-0.7738584371,3.9334929405 \backslash \mathrm{H}, 1.7577926639,-0.0442170$ $742,3.8747115992 \backslash \mathrm{Si}, 0.6915123994,2.9503246052,-1.0790834737 \backslash \mathrm{H}, 1.311961$ $8206,3.6582077463,0.061448491 \backslash \mathrm{H}, 1.6373524876,2.8370899988,-2.210032265$ $2 \backslash \mathrm{H},-0.5419606207,3.6502810642,-1.5096165528 \backslash \mathrm{Na},-0.912122615,-1.973001$ $773,0.6189103873 \backslash 0,-2.7197689739,-0.9772884489,0.0736056877 \backslash \mathrm{C},-3.05262$ $38377,0.0407750442,-0.6156076281 \backslash 0,-4.0116450294,0.9022380208,-0.09765$ $31729 \backslash \mathrm{H},-1.9644126575,0.8990546208,-0.7508830211 \backslash \mathrm{H},-0.9951014172,1.163$ $1381151,-0.6951484783 \backslash \mathrm{H},-3.2329748016,-0.0525495784,-1.6965421803 \backslash \mathrm{H},-4$ $.032515321,0.7317578666,0.8572945623 \backslash \mathrm{Si}, 1.1350538836,-2.107600369,-2.3$ $175399087 \backslash \mathrm{H}, 3.6068665329,0.3197944429,0.3074827381 \backslash \mathrm{H}, 2.5459517833,-2.5$ $734835393,-2.3839691649 \backslash \mathrm{H}, 0.2424025527,-3.2867770941,-2.4942405303 \backslash \mathrm{H}, 0$ $.8955151083,-1.1570621817,-3.4356028364 \backslash \backslash$ Version=DEC-AXP-OSF / 1-G03RevB $.03 \backslash$ State $=1-A \backslash H F=-1771.4806729 \backslash \mathrm{RMSD}=4.295 \mathrm{e}-09 \backslash \mathrm{RMSF}=1.970 \mathrm{e}-06 \backslash \mathrm{Dipole}=0$. $6490138,-1.0330044,0.5703593 \backslash \mathrm{PG}=\mathrm{C} 01[\mathrm{X}(\mathrm{C} 1 \mathrm{H} 14 \mathrm{Al} 1 \mathrm{Na} 106 \mathrm{Si} 3)] \backslash \backslash @$

\section{$2 \cdot \mathrm{CH}_{2}(\mathrm{OH})_{2}$}

$1 \backslash 1 \backslash \mathrm{GINC}-\mathrm{SC} 20 \backslash \mathrm{FOpt} \backslash \mathrm{RB} 3 \mathrm{LYP} \backslash 6-31+\mathrm{G}(\mathrm{d}, \mathrm{p}) \backslash \mathrm{C} 1 \mathrm{H} 14 \mathrm{Al} 1 \mathrm{Na} 106 \mathrm{Si} \backslash \mathrm{BUC5} 62 \backslash 25-\mathrm{Jan}-2$

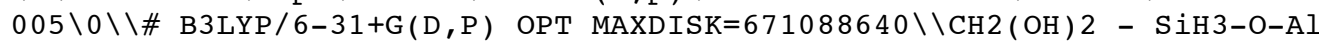
$\mathrm{OH}(\mathrm{OSiH} 3)-\mathrm{ONa}-\mathrm{SiH} 3 \backslash \backslash 0,1 \backslash \mathrm{Al},-1.2120075563,-0.2233976138,-0.1984783345 \backslash \mathrm{O}$ $,-0.9103245625,-0.3672948424,1.5675998091 \backslash 0,0.2498611156,-0.8528911637$ $,-0.9903729044 \backslash 0,-2.6253908993,-0.9704441492,-0.8572589577 \backslash 0,-1.082871$ $0363,1.5689437855,-0.2659650938 \backslash \mathrm{Si},-1.0295121897,-1.5215301542,2.73920$ $17282 \backslash \mathrm{H},-0.4631509531,-2.8282857027,2.311483546 \backslash \mathrm{H},-0.2600992012,-1.049$ $1798689,3.9295988496 \backslash \mathrm{H},-2.440221093,-1.7322170918,3.1628308731 \backslash \mathrm{Si}, 0.55$ $01508403,-1.9389882551,-2.2077426394 \backslash \mathrm{H}, 0.19541341,-3.3259889491,-1.804$ $9074104 \backslash \mathrm{H},-0.2010180141,-1.5964958284,-3.4444443775 \backslash \mathrm{H}, 2.0055138993,-1$. $896273943,-2.5153219332 \backslash \mathrm{Na}, 0.3425177697,1.5184027409,1.4978977195 \backslash \mathrm{O}, 2$. $0898860504,0.5360410015,0.4423576975 \backslash C, 3.4366785917,0.6080136101,0.014$ $4717952 \backslash 0,4.120322126,-0.6081805535,0.1681767274 \backslash \mathrm{H}, 3.4984354663,0.8444$ $49442,-1.0518796058 \backslash \mathrm{H}, 1.5657503481,-0.0662133338,-0.1554428611 \backslash \mathrm{H}, 3.896$ $3618098,1.4112202865,0.604536677 \backslash \mathrm{H}, 4.1088341831,-0.858394366,1.1027838$ $601 \backslash \mathrm{Si},-1.4536901259,2.7773012373,-1.3253422013 \backslash \mathrm{H},-3.5285663355,-0.785$ $9282802,-0.5912087018 \backslash \mathrm{H},-2.9184860351,3.0329339843,-1.3801064319 \backslash \mathrm{H},-0$. $7873582816,4.0266634444,-0.8496558268 \backslash \mathrm{H},-0.9722196844,2.4930151601,-2$. $7036886442 \backslash \backslash$ Version=DEC-AXP-OSF / 1-G03RevB.03 $\backslash$ State $=1-\mathrm{A} \backslash \mathrm{HF}=-1771.551061$ $2 \backslash \mathrm{RMSD}=2.279 \mathrm{e}-09 \backslash \mathrm{RMSF}=2.681 \mathrm{e}-06 \backslash \mathrm{Dipole}=0.8808047,1.9165345,1.9453714 \backslash \mathrm{P}$ $\mathrm{G}=\mathrm{C} 01[\mathrm{X}(\mathrm{C} 1 \mathrm{H} 14 \mathrm{~A} 11 \mathrm{Na} 1 \mathrm{O} 6 \mathrm{Si3})] \backslash \backslash @$ 


\section{$2 \cdot \mathrm{CH}_{2} \mathrm{O}$}

$1 \backslash 1 \backslash$ GINC-SC $4 \backslash F O p t \backslash R B 3 L Y P \backslash 6-31+G(d, p) \backslash C 1 H 12 A 11 N a 105 S i 3 \backslash B U C 562 \backslash 25-J a n-20$ $05 \backslash 0 \backslash \backslash \#$ B3LYP/6-31+G(D,P) OPT MAXDISK=671088640 \CH2O SiH3-O-AlOH(OSiH $3)-\mathrm{ONa}-\mathrm{SiH} 3 \backslash \backslash 0,1 \backslash \mathrm{Al}, 1.1635807051,0.0162034283,-0.2855637807 \backslash 0,-0.02556$ $10472,-1.3287789026,-0.5592875878 \backslash 0,1.7156319317,-0.0146184513,1.35302$ $00959 \backslash 0,2.4723576963,0.0520892379,-1.4296153192 \backslash 0,-0.0484964039,1.3511$ $986748,-0.5008653219 \backslash \mathrm{Si}, 0.0779254891,-2.968923577,-0.6942777404 \backslash \mathrm{H}, 0.86$ $08104988,-3.5873407021,0.4075797693 \backslash \mathrm{H},-1.3116041786,-3.5201456745,-0.6$ $409707754 \backslash \mathrm{H}, 0.6736229096,-3.3865625978,-1.9929258956 \backslash \mathrm{Si}, 2.5215037011,-$ $0.0390137575,2.7639294499 \backslash \mathrm{H}, 3.4004764426,-1.237850355,2.8841436862 \backslash \mathrm{H}, 3$ $.3776937549,1.1695674175,2.9386360036 \backslash \mathrm{H}, 1.5655510891,-0.0740563719,3.9$ $117710476 \backslash \mathrm{Na},-1.8038748306,-0.0047029473,-0.4960948083 \backslash 0,-4.0667615999$ $,-0.0242104907,-0.4577064221 \backslash \mathrm{C},-5.2749259531,-0.0375491765,-0.33086356$ $4 \backslash \mathrm{H},-5.9421093573,-0.0208833781,-1.2096617912 \backslash \mathrm{H},-5.7464023579,-0.06674$ $9582,0.6664312384 \backslash \mathrm{Si}, 0.0253446842,2.9972679816,-0.5634109207 \backslash \mathrm{H}, 2.32709$ $88776,0.0708949886,-2.3785266634 \backslash \mathrm{H}, 0.6138476295,3.4824167828,-1.841705$ $4806 \backslash \mathrm{H},-1.3739872921,3.5203619141,-0.4874897789 \backslash \mathrm{H}, 0.7964328158,3.58066$ $08651,0.5655374624 \backslash \backslash$ Version=DEC-AXP-OSF $/ 1-G 03 R e v B .03 \backslash$ State $=1-A \backslash H F=-169$ $5.0873879 \backslash \mathrm{RMSD}=4.547 \mathrm{e}-09 \backslash \mathrm{RMSF}=2.837 \mathrm{e}-06 \backslash \mathrm{Dipole}=-5.2100989,-0.0272673,-$

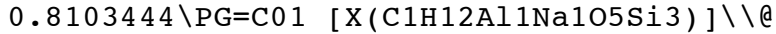

\section{TS $\left(2, \mathrm{CH}_{2} \mathrm{O}\right)$}

$1 \backslash 1 \backslash G I N C-S C 62 \backslash F T S \backslash R B 3 L Y P \backslash 6-31+G(d, p) \backslash C 1 H 14 A 11 N a 105 S i 3 \backslash B U C 562 \backslash 30-J a n-20$ $05 \backslash 0 \backslash \backslash \#$ B $3 L Y P / 6-31+G(D, P) \quad O P T=(T S, C A L C F C$, NOEIGENTEST, LOOSE $) \quad$ MAXDISK $=26$

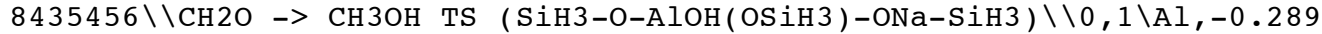
$2051143,0.5171021923,-0.7386930332 \backslash 0,1.4444513623,0.1687468172,-0.8688$ $072874 \backslash 0,-0.6169421467,1.0992084242,0.9637164374 \backslash 0,-0.9924540708,1.780$ $5818907,-1.6846727007 \backslash 0,-0.902458036,-1.1415694717,-0.8673727634 \backslash \mathrm{Si}, 2$. $8827389129,0.9682442227,-1.0237119443 \backslash \mathrm{H}, 3.0671792341,1.990459255,0.040$ $6223616 \backslash \mathrm{H}, 3.981587498,-0.0320175356,-0.9164530944 \backslash \mathrm{H}, 2.9887712135,1.639$ $0670307,-2.3473439616 \backslash \mathrm{Si},-1.3917690731,2.5256028769,1.4558344967 \backslash \mathrm{H},-0$. $646520786,3.7084154933,0.9710364255 \backslash \mathrm{H},-2.7877792,2.5476183535,0.966388$ $5453 \backslash \mathrm{H},-1.377780308,2.4944660351,2.9397577658 \backslash \mathrm{Na}, 1.1355724718,-2.03298$ $94058,-0.221687105 \backslash 0,1.1972300413,-2.1630175394,1.9025695263 \backslash \mathrm{C}, 0.80568$ $00577,-1.4776288498,2.9012888625 \backslash \mathrm{H}, 1.5533672085,-0.9335220974,3.506200$ $6704 \backslash \mathrm{H}, 0.1254793993,-0.2788999292,2.5401343167 \backslash \mathrm{H},-0.2109073281,0.34233$ $95294,1.8893799611 \backslash \mathrm{H},-0.0478104508,-1.8414635611,3.5014696177 \backslash \mathrm{Si},-2.34$ $05581342,-1.9426423571,-1.0166558651 \backslash \mathrm{H},-1.012425788,1.8434825024,-2.64$ $14877011 \backslash \mathrm{H},-2.9513681682,-1.7118896589,-2.3534986025 \backslash \mathrm{H},-2.0757641225,-$ $3.4011993444,-0.8692303843 \backslash \mathrm{H},-3.3161205297,-1.5289953737,0.0268591722 \backslash$ VVersion=DEC-AXP-OSF $/ 1-G 03 R e v B .03 \backslash$ State $=1-A \backslash H F=-1696.2352507 \backslash$ RMSD $=4.35$ $1 \mathrm{e}-09 \backslash \mathrm{RMSF}=7.593 \mathrm{e}-06 \backslash \mathrm{Dipole}=0.3121392,-0.5310178,-0.4893359 \backslash \mathrm{PG}=\mathrm{C} 01 \quad[\mathrm{X}($ C1H14Al1Na105Si3) ] \\@

\section{$2 \cdot \mathrm{CH}_{3} \mathrm{OH}$}

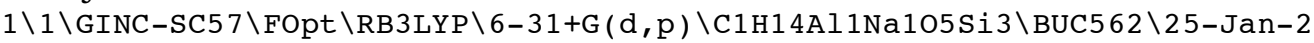
$005 \backslash 0 \backslash \backslash \#$ B $3 L Y P / 6-31+G(D, P)$ OPT MAXDISK $=671088640 \backslash \backslash \mathrm{C} 3 \mathrm{OH}$ SiH3-O-AlOH (OSi H3) -ONa-SiH $\backslash \backslash \backslash 0,1 \backslash \mathrm{Al},-1.0031338347,0.0462258041,0.1150011205 \backslash 0,-0.4154$ $770201,1.6131600499,-0.5403699086 \backslash 0,0.1934551679,-0.4602695157,1.32893$ $73382 \backslash 0,-2.5939655289,-0.0210598994,0.7905592478 \backslash 0,-0.6500680502,-0.91$ $89515482,-1.3585602899 \backslash \mathrm{Si},-0.5061052274,3.21060516,-0.1444978436 \backslash \mathrm{H},-0$. $2073233857,3.4586677441,1.2915561006 \backslash \mathrm{H}, 0.5032594568,3.9474130569,-0.96$ $25488976 \backslash \mathrm{H},-1.845956196,3.7810096233,-0.4517576378 \backslash \mathrm{Si}, 0.1476853008,-0$. $9814472144,2.898445764 \backslash \mathrm{H},-0.4336827491,0.0412746781,3.8079645047 \backslash \mathrm{H},-0$. $6237799744,-2.2441774491,3.0457184349 \backslash \mathrm{H}, 1.549944649,-1.244056258,3.333$ $9678588 \backslash \mathrm{Na}, 1.0651307405,0.4897290758,-1.8589697551 \backslash 0,2.435142997,-0.20$ $29083076,-0.2276136872 \backslash \mathrm{C}, 3.7404558479,-0.2617607933,0.3433690633 \backslash \mathrm{H}, 3.9$ $247495717,-1.2371021301,0.8087776593 \backslash \mathrm{H}, 4.463510937,-0.1181155894,-0.46$ $35999344 \backslash \mathrm{H}, 1.7474026792,-0.3581468524,0.4678648316 \backslash \mathrm{H}, 3.8858895955,0.52$ $67766179,1.0918611372 \backslash \mathrm{Si},-1.007083337,-2.4137831203,-1.9562415945 \backslash \mathrm{H},-3$ $.4059456874,0.193381984,0.326559343 \backslash \mathrm{H},-2.4030668947,-2.4831471471,-2.4$ $677841548 \backslash \mathrm{H},-0.0871626427,-2.6864166823,-3.1006757018 \backslash \mathrm{H},-0.8219275752$, $-3.4897659103,-0.9459753488 \backslash \backslash$ Version=DEC-AXP-OSF / 1-G03RevB.03\State=1$\mathrm{A} \backslash \mathrm{HF}=-1696.3220009 \backslash \mathrm{RMSD}=4.498 \mathrm{e}-09 \backslash \mathrm{RMSF}=1.007 \mathrm{e}-04 \backslash \mathrm{Dipole}=1.7364782,0.51$ $17683,-1.7992433 \backslash \mathrm{PG}=\mathrm{C} 01 \quad[\mathrm{X}(\mathrm{C} 1 \mathrm{H} 14 \mathrm{Al} 1 \mathrm{Na} 105 \mathrm{Si3})] \backslash \backslash @$ 


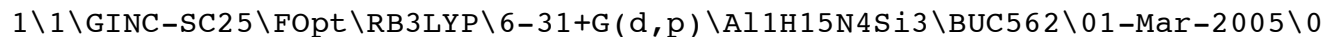
$\backslash \backslash \#$ B3LYP/6-31+G(D,P) OPT MAXDISK=268435456 \SiH3-NH-AlNH2 (NHSiH3) -NH2 $-\mathrm{SiH} 3 \backslash \backslash 0,1 \backslash \mathrm{Al},-0.04934359,-0.4745486575,-0.522929322 \backslash \mathrm{N}, 1.6880179891,-0$ $.4696007528,-1.129503266 \backslash \mathrm{N},-0.1897212105,1.3774324401,0.3762637853 \backslash \mathrm{N},-$ $1.3227125241,-0.3375068467,-1.8241402788 \backslash \mathrm{N},-0.4221579543,-1.5612731562$ $, 0.9180112022 \backslash \mathrm{Si}, 3.1769783273,-0.2300297946,-0.2886389672 \backslash \mathrm{H}, 3.76135925$ $77,-1.4416748868,0.3609058268 \backslash \mathrm{H}, 4.2315001386,0.3282772315,-1.182715863$ $3 \backslash \mathrm{H}, 2.9339637318,0.7546930669,0.8121472227 \backslash \mathrm{Si},-1.2212278155,2.56483265$ $85,-0.5334012597 \backslash \mathrm{H},-2.628272233,2.1512073266,-0.4023096233 \backslash \mathrm{H},-1.028490$ $4488,3.8897961254,0.116736818 \backslash \mathrm{H},-0.731751597,2.6331465207,-1.922084770$ $6 \backslash \mathrm{H}, 0.7673784422,1.7157591851,0.4949853593 \backslash \mathrm{Si},-1.7185439845,-1.5745639$ $048,2.0553505415 \backslash \mathrm{H},-2.934830398,-2.3442231198,1.6540726092 \backslash \mathrm{H},-1.297009$ $4502,-2.1042432497,3.383595565 \backslash \mathrm{H},-2.1702618761,-0.1592958653,2.2439903$ $562 \backslash \mathrm{H},-2.1406358608,-0.9357419612,-1.8245803244 \backslash \mathrm{H},-1.1276172911,-0.054$ $6024487,-2.7771404489 \backslash \mathrm{H},-0.5528257464,1.2186927046,1.3186198118 \backslash \mathrm{H}, 0.16$ $38410064,-2.3918677712,0.9561778938 \backslash \mathrm{H}, 1.8002435128,-0.7974975298,-2.08$ $50837489 \backslash \backslash$ Version $=\mathrm{DEC}-\mathrm{AXP}-\mathrm{OSF} / 1-\mathrm{G} 03$ RevB.03 $\backslash$ State $=1-\mathrm{A} \backslash \mathrm{HF}=-1339.18031 \backslash \mathrm{RM}$ $\mathrm{SD}=8.402 \mathrm{e}-09 \backslash \mathrm{RMSF}=1.913 \mathrm{e}-05 \backslash \mathrm{Dipole}=-0.2362233,0.9330753,-0.2255902 \backslash \mathrm{PG}=$ $\mathrm{C} 01[\mathrm{X}(\mathrm{Al} 1 \mathrm{H} 15 \mathrm{~N} 4 \mathrm{Si3})] \backslash \backslash \mathrm{Q}$

\section{$3 \cdot \mathrm{CO}_{2}$}

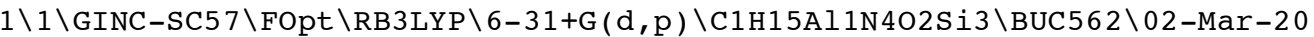

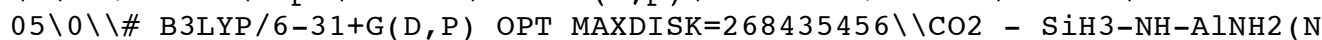
HSiH3 )-NH $2-\mathrm{SiH} 3 \backslash \backslash 0,1 \backslash \mathrm{Al},-0.5380760311,-0.7906453752,-0.8568758138 \backslash \mathrm{N}, 1$. $4830267332,-0.6980804547,-0.4488718121 \backslash N,-0.8200103571,0.7445660189,-1$ $.8359390681 \backslash \mathrm{N},-0.6070975083,-2.3664727762,-1.7716056519 \backslash \mathrm{N},-1.236239570$ $2,-0.8092615325,0.8533147852 \backslash \mathrm{Si}, 2.5124151497,-0.0226841869,-1.77479238$ $51 \backslash \mathrm{H}, 2.4033101294,1.4463999689,-1.7173730362 \backslash \mathrm{H}, 3.9180853586,-0.4410343$ $669,-1.5421816534 \backslash \mathrm{H}, 2.0305763125,-0.5818364581,-3.053030877 \backslash \mathrm{Si},-0.9132$ $828496,2.4079725627,-1.374582449 \backslash \mathrm{H},-2.2854897073,2.8958356212,-1.04165$ $48737 \backslash \mathrm{H},-0.0799621772,2.5937993617,-0.1520221175 \backslash \mathrm{H},-0.3865636905,3.318$ $9414113,-2.4324322243 \backslash \mathrm{H}, 1.6061321797,-0.1818441907,0.4260243199 \backslash 0,1.34$ $48873027,1.076471131,2.216858593 \backslash \mathrm{C}, 0.3141963334,1.4803581516,2.6048931$ $951 \backslash 0,-0.689544718,1.9065950376,3.016703385 \backslash \mathrm{Si},-1.0911422075,-2.053717$ $9009,2.0480921772 \backslash \mathrm{H}, 0.235776186,-2.7071486894,1.8571677863 \backslash \mathrm{H},-1.137339$ $4244,-1.5047319587,3.4356038385 \backslash \mathrm{H},-2.124869036,-3.1301278244,1.9864442$ $385 \backslash \mathrm{H},-1.2546971511,-3.1013356039,-1.5136414975 \backslash \mathrm{H},-0.3564451259,-2.483$ $3284716,-2.7459862528 \backslash \mathrm{H},-2.050187397,-0.2115595289,0.9791438074 \backslash \mathrm{H}, 1.71$ $65103577,-1.6731792466,-0.2512745678 \backslash \mathrm{H},-1.2173834699,0.555616159,-2.75$ $35868796 \backslash \backslash$ Version=DEC-AXP-OSF $/ 1-G 03$ RevB.03 \State $=1-A \backslash H F=-1527.7744583 \backslash$ RMSD $=2.947 \mathrm{e}-09 \backslash \mathrm{RMSF}=2.342 \mathrm{e}-05 \backslash \mathrm{Dipole}=1.1029388,-0.1276472,-0.439875 \backslash \mathrm{PG}$ $=\mathrm{C} 01[\mathrm{X}(\mathrm{C} 1 \mathrm{H} 15 \mathrm{Al} 1 \mathrm{~N} 4 \mathrm{O} 2 \mathrm{Si3})] \backslash \backslash @$

\section{$\operatorname{TS}\left(3, \mathrm{CO}_{2}\right)$}

$1 \backslash 1 \backslash G I N C-S C 24 \backslash F T S \backslash R B 3 L Y P \backslash 6-31+G(d, p) \backslash C 1 H 17 A l 1 N 402 S i 3 \backslash B U C 562 \backslash 03-M a r-200$ $5 \backslash 0 \backslash \backslash \#$ B3LYP $/ 6-31+G(D, P) \quad O P T=(T S, C A L C F C$, NOEIGENTEST $)$ MAXDISK $=268435456$

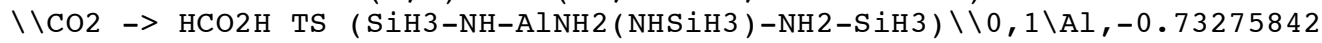
$2,0.0242906987,0.9072902515 \backslash N,-0.011809535,1.1093475685,-0.6223336791 \backslash$ $\mathrm{N}, 0.4502452606,-1.4859083719,0.9061467969 \backslash \mathrm{N},-0.4909108303,0.9487682663$ $, 2.443595207 \backslash \mathrm{N},-2.4777775987,-0.2778847844,0.430798799 \backslash \mathrm{Si}, 0.0301085022$ $, 2.9022515012,-0.2925653679 \backslash \mathrm{H}, 1.1502211275,3.163795787,0.627066078 \backslash \mathrm{H}, 0$ $.2416863103,3.582972636,-1.5929181236 \backslash \mathrm{H},-1.2788552099,3.2869312989,0.2$ $71366131 \backslash \mathrm{Si}, 0.1691466322,-2.8475538051,-0.2027739367 \backslash \mathrm{H}, 1.4131790417,-3$ $.644599456,-0.3484050386 \backslash \mathrm{H},-0.9177228308,-3.7333386208,0.2832471074 \backslash \mathrm{H}$, $-0.2017966988,-2.2722003517,-1.518418444 \backslash \mathrm{H}, 0.9495292605,0.8071475743,-$ $0.8614956638 \backslash 0,2.7610622534,0.5280409367,-1.1802647731 \backslash \mathrm{C}, 3.5370916145$, $-0.048568657,-0.4510807346 \backslash 0,4.656115792,-0.3199283882,-0.1470436919 \backslash \mathrm{H}$ $, 2.6698084989,-0.7807688151,0.5796203815 \backslash \mathrm{H}, 1.8005038484,-1.0590844569$, $0.7151614562 \backslash \mathrm{Si},-3.3645116377,-0.2882839763,-1.052815618 \backslash \mathrm{H},-4.59162255$ $13,0.5562704748,-1.0033142339 \backslash \mathrm{H},-2.4732130369,0.2685683714,-2.11592206$ $98 \backslash \mathrm{H},-3.791160234,-1.6420509896,-1.5102398977 \backslash \mathrm{H},-1.2098928364,1.500973$ $3079,2.8928413486 \backslash \mathrm{H}, 0.3332693571,0.9250574922,3.030253789 \backslash \mathrm{H},-3.0524531$ $476,-0.5383759276,1.2307637851 \backslash \mathrm{H},-0.5813028647,0.9211362157,-1.4518172$ $644 \backslash \mathrm{H}, 0.5310673712,-1.821752896,1.8691085681 \backslash \backslash$ Version=DEC-AXP-OSF $/ 1-G 0$ 3 RevB.03 $\backslash$ State $=1-A \backslash H F=-1528.912452 \backslash \mathrm{RMSD}=9.939 \mathrm{e}-09 \backslash \mathrm{RMSF}=4.814 \mathrm{e}-06 \backslash \mathrm{Dipol}$ $\mathrm{e}=-1.6044614,0.321306,0.5585661 \backslash \mathrm{PG}=\mathrm{C} 01 \quad[\mathrm{X}(\mathrm{C} 1 \mathrm{H} 17 \mathrm{Al} 1 \mathrm{~N} 4 \mathrm{O} 2 \mathrm{Si} 3)] \backslash \backslash @$ 


\section{$3 \cdot \mathrm{HCO}_{2} \mathrm{H}$}

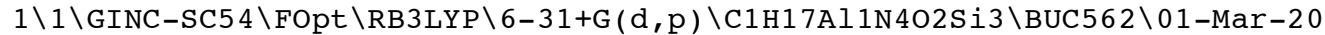
$05 \backslash 0 \backslash \backslash \#$ B $3 L Y P / 6-31+G(D, P)$ OPT MAXDISK=268435456\\HCO2H - siH3-NH-AlNH2

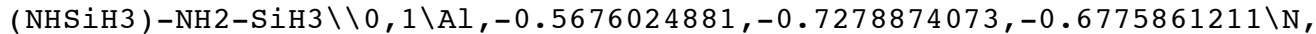
$1.2013301601,-0.9267913765,-0.0437854131 \backslash \mathrm{N},-0.6977100105,1.3090033441$, $-0.8441871901 \backslash \mathrm{N},-1.0216514541,-1.1441776797,-2.3931441859 \backslash \mathrm{N},-1.7956651$ $673,-1.2509571275,0.5731937192 \backslash \mathrm{Si}, 2.5156075162,-1.3402481589,-1.131582$ $2864 \backslash \mathrm{H}, 2.4585415704,-0.3837103913,-2.2701824386 \backslash \mathrm{H}, 3.8282677485,-1.1845$ $839298,-0.4464215138 \backslash \mathrm{H}, 2.4516472349,-2.7227175608,-1.6786093025 \backslash \mathrm{Si},-2$. $2571337095,1.8811052128,-1.5891376096 \backslash \mathrm{H},-3.3350790806,0.9564664913,-1$. $1951943566 \backslash \mathrm{H},-2.5320406109,3.2394943335,-1.0609302876 \backslash \mathrm{H},-2.0483577022$, $1.9293044754,-3.0489130191 \backslash \mathrm{H}, 1.5135242525,0.5834359715,0.6756631169 \backslash 0$, $1.488545608,1.5459845557,1.016284507 \backslash \mathrm{C}, 2.4026293189,1.8066282031,1.958$ $4843477 \backslash \mathrm{O}, 2.5213414015,2.8839167738,2.4904212289 \backslash \mathrm{H}, 3.0453130765,0.9415$ $459209,2.2057215975 \backslash \mathrm{H},-0.5276022499,1.7165686714,0.0805758993 \backslash \mathrm{Si},-1.65$ $52897212,-1.8107555155,2.2043441546 \backslash \mathrm{H},-2.1190085514,-3.2171183196,2.38$ $81480804 \backslash \mathrm{H},-0.2155692481,-1.7537580366,2.5853411997 \backslash \mathrm{H},-2.4177068247,-0$ $.9833339569,3.1838005655 \backslash \mathrm{H},-1.5202686485,-2.0007034584,-2.6067187642 \backslash \mathrm{H}$ $,-0.5017242496,-0.8682408861,-3.2180531705 \backslash \mathrm{H},-2.7424493521,-1.31826457$ $76,0.2066166719 \backslash \mathrm{H}, 1.2032450197,-1.5180884834,0.7893786393 \backslash \mathrm{H}, 0.09452607$ $71,1.6362985192,-1.4014334489 \backslash \backslash$ Version=DEC-AXP-OSF / 1-G03RevB.03\State= $1-\mathrm{A} \backslash \mathrm{HF}=-1528.9669261 \backslash \mathrm{RMSD}=4.752 \mathrm{e}-09 \backslash \mathrm{RMSF}=1.791 \mathrm{e}-04 \backslash \mathrm{Dipole}=-0.861745,-0$ $.5280897,-1.569716 \backslash \mathrm{PG}=\mathrm{C} 01 \quad[\mathrm{X}(\mathrm{C} 1 \mathrm{H} 17 \mathrm{Al} 1 \mathrm{~N} 402 \mathrm{Si} 3)] \backslash \backslash \mathrm{a}$

\section{$\operatorname{TS}\left(3, \mathrm{HCO}_{2} \mathrm{H}\right)$}

$1 \backslash 1 \backslash$ GINC-SC4 $6 \backslash F T S \backslash R B 3 L Y P \backslash 6-31+G(d, p) \backslash C 1 H 19 A 11 N 402 S i 3 \backslash B U C 562 \backslash 02-M a r-200$ $5 \backslash 0 \backslash \backslash \#$ B3LYP/6-31+G(D,P) OPT=(TS, CALCFC, NOEIGENTEST) MAXDISK=268435456 $\backslash \backslash \mathrm{HCO} 2 \mathrm{H} \rightarrow \mathrm{CH} 2(\mathrm{OH}) 2 \mathrm{TS}(\mathrm{SiH} 3-\mathrm{NH}-\mathrm{AlNH} 2(\mathrm{NHSiH} 3)-\mathrm{NH} 2-\mathrm{SiH} 3) \backslash \backslash 0,1 \backslash \mathrm{Al},-0.573$ $1077319,-0.6276864231,-0.8029982716 \backslash \mathrm{N}, 1.2499776156,-1.0896129578,-0.11$ $35872757 \backslash \mathrm{N},-0.5635706337,1.2737615477,-0.4995531568 \backslash \mathrm{N},-0.6340300687,-0$ $.9677035499,-2.5835089829 \backslash \mathrm{N},-1.7287804118,-1.5982667667,0.2323319851 \backslash \mathrm{S}$ $i, 2.4393532279,-1.6458271338,-1.3601221311 \backslash \mathrm{H}, 1.9227399011,-2.879243574$ $8,-1.9876351982 \backslash \mathrm{H}, 2.6225446757,-0.5505829911,-2.3312310514 \backslash \mathrm{H}, 3.7196456$ $102,-1.938987904,-0.6688350153 \backslash \mathrm{Si},-1.9436159545,2.262565692,-1.0267309$ $374 \backslash \mathrm{H},-1.8514008774,2.5706392449,-2.4777660077 \backslash \mathrm{H},-3.1828471578,1.49918$ $23811,-0.7555144118 \backslash \mathrm{H},-1.9654200045,3.5417312643,-0.2724847187 \backslash \mathrm{H}, 1.631$ $1326674,-0.2347134988,0.3813616865 \backslash 0,2.0738737379,1.2713626531,0.98304$ $12839 \backslash \mathrm{C}, 1.4781294683,1.7413204114,1.9943052811 \backslash 0,1.6615058179,3.063526$ $2947,2.3283429886 \backslash \mathrm{H},-0.025772267,1.7201725648,1.7014825066 \backslash \mathrm{H},-0.358600$ $7872,1.5163785127,0.823668633 \backslash \mathrm{H}, 1.3352179574,1.1589905777,2.9129855938$ $\backslash \mathrm{H}, 2.0534683626,3.4879457774,1.5482638468 \backslash \mathrm{Si},-1.7565086449,-2.01779696$ $86,1.9095260855 \backslash \mathrm{H},-2.5990886108,-1.1376528241,2.7706580094 \backslash \mathrm{H},-0.357472$ $1339,-1.9156055355,2.4239012508 \backslash \mathrm{H},-2.2291776838,-3.414466115,2.1298750$ $695 \backslash \mathrm{H},-0.9157731633,-1.8513723246,-2.9891572012 \backslash \mathrm{H},-0.589181603,-0.2657$ $08265,-3.3107437915 \backslash \mathrm{H}, 1.1341167032,-1.8015733261,0.6112036569 \backslash \mathrm{H},-2.579$ $9716708,-1.8548644829,-0.2627806072 \backslash \mathrm{H}, 0.3200510314,1.6701898004,-0.832$ $3808114 \backslash \backslash$ Version=DEC-AXP-OSF / 1-G03RevB.03\State $=1-\mathrm{A} \backslash \mathrm{HF}=-1530.0941598 \backslash \mathrm{R}$ $\mathrm{MSD}=9.340 \mathrm{e}-09 \backslash \mathrm{RMSF}=1.442 e-05 \backslash \mathrm{Dipole}=0.1267788,-0.5074039,-1.1535977 \backslash \mathrm{PG}$ $=\mathrm{C} 01[\mathrm{X}(\mathrm{C} 1 \mathrm{H} 19 \mathrm{~A} 11 \mathrm{~N} 4 \mathrm{O} 2 \mathrm{Si3})] \backslash \backslash \mathrm{Q}$ 


\section{$3 \cdot \mathrm{CH}_{2}(\mathrm{OH})_{2}$}

$1 \backslash 1 \backslash$ GINC-SC45 $\backslash F O p t \backslash R B 3 L Y P \backslash 6-31+G(d, p) \backslash C 1 H 19 A 11 N 4 O 2 S i 3 \backslash B U C 562 \backslash 02-M a r-20$ $05 \backslash 0 \backslash \backslash \#$ B3LYP $/ 6-31+G(D, P) \quad O P T$ MAXDISK=268435456 \CH2(OH) 2 - SiH3-NH-Al

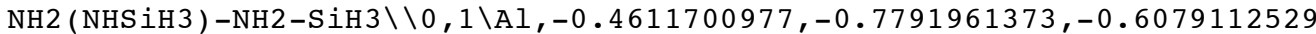
$\backslash \mathrm{N}, 1.3792869147,-0.904222189,-0.2656162396 \backslash \mathrm{N},-0.9998328607,0.998323319$ $9,0.2294233202 \backslash \mathrm{N},-0.866832903,-0.4932226597,-2.3641667785 \backslash \mathrm{N},-1.4753758$ $841,-1.9845330431,0.3437430761 \backslash \mathrm{Si}, 2.047819805,-1.7899625553,1.08732932$ $28 \backslash \mathrm{H}, 1.7222384777,-3.2467936145,1.0976481115 \backslash \mathrm{H}, 3.5327535306,-1.6731669$ $431,1.0954484706 \backslash \mathrm{H}, 1.5155127551,-1.2019751466,2.3448486244 \backslash \mathrm{Si},-2.00199$ $4898,2.0495105356,-0.8644389791 \backslash \mathrm{H},-3.1899105826,1.2935959337,-1.300215$ $3426 \backslash \mathrm{H},-2.4380503567,3.2133704136,-0.0453333816 \backslash \mathrm{H},-1.1508810592,2.5201$ $208419,-1.9705724089 \backslash \mathrm{H}, 1.830603891,0.8723150638,0.0142797301 \backslash 0,1.74762$ $09346,1.8481271575,0.2019697234 \backslash \mathrm{C}, 2.9658763795,2.3819018786,0.66284906$ $29 \backslash 0,3.9491295194,2.4455120184,-0.3476617315 \backslash \mathrm{H}, 2.7313559316,3.37816467$ $37,1.0568303933 \backslash \mathrm{H},-0.1290050414,1.4937943203,0.4763998681 \backslash \mathrm{H}, 3.40704519$ $7,1.7629350703,1.451322491 \backslash \mathrm{H}, 3.6160312612,3.0104243813,-1.0590211171 \backslash \mathrm{S}$ $i,-3.0446194503,-1.9223064466,1.0618830256 \backslash \mathrm{H},-3.3276117309,-0.50196958$ $27,1.4344997481 \backslash \mathrm{H},-4.1735879582,-2.352332261,0.183144619 \backslash \mathrm{H},-3.12680130$ $28,-2.7489906406,2.2990423907 \backslash \mathrm{H},-1.5665754422,-1.0525815174,-2.8374497$ $285 \backslash \mathrm{H},-0.2258227911,-0.1210470634,-3.0547053005 \backslash \mathrm{H},-1.0903208348,-2.926$ $9818276,0.332652657 \backslash \mathrm{H}, 1.9040724103,-1.077777185,-1.1230003493 \backslash \mathrm{H},-1.492$ $6902547,0.8121207216,1.1049576817 \backslash \backslash$ Version=DEC-AXP-OSF / 1-G03RevB.03\St ate $=1-\mathrm{A} \backslash \mathrm{HF}=-1530.1636914 \backslash \mathrm{RMSD}=9.986 \mathrm{e}-09 \backslash \mathrm{RMSF}=9.090 \mathrm{e}-06 \backslash \mathrm{Dipole}=-0.34717$ $25,0.8470251,-0.2896385 \backslash \mathrm{PG}=\mathrm{C} 01 \quad[\mathrm{X}(\mathrm{C} 1 \mathrm{H} 19 \mathrm{~A} 11 \mathrm{~N} 4 \mathrm{O} 2 \mathrm{Si3})] \backslash \backslash @$

\section{$3 \cdot \mathrm{CH}_{2} \mathrm{O}$}

$1 \backslash 1 \backslash G I N C-S C 18 \backslash F O p t \backslash R B 3 L Y P \backslash 6-31+G(d, p) \backslash C 1 H 17 A 11 N 401 S i 3 \backslash B U C 562 \backslash 02-M a r-20$ $05 \backslash 0 \backslash \backslash \#$ B $3 L Y P / 6-31+G(D, P)$ OPT MAXDISK $=268435456 \backslash \backslash \mathrm{CH} 2 \mathrm{O}$ SiH3-NH-AlNH $2(\mathrm{NH}$ $\mathrm{SiH} 3)-\mathrm{NH} 2-\mathrm{SiH} 3 \backslash \backslash 0,1 \backslash \mathrm{Al},-0.6951041098,-0.2670110871,-0.3536983754 \backslash \mathrm{N}, 1.2$ $805743138,-0.7755572939,-0.1508441453 \backslash \mathrm{N},-0.5931007289,1.3637931795,-1$. $2065465098 \backslash \mathrm{N},-1.3056550856,-1.6857341245,-1.3227337938 \backslash \mathrm{N},-1.2727484327$ $,-0.1770419926,1.4059526674 \backslash \mathrm{Si}, 2.352786318,-0.2067894513,-1.4922040637$ $\backslash \mathrm{H}, 1.6722252925,-0.470616975,-2.7752628346 \backslash \mathrm{H}, 2.6213799527,1.2271613405$ $,-1.2746342917 \backslash \mathrm{H}, 3.6227097437,-0.9755355904,-1.4363560718 \backslash \mathrm{Si},-1.296350$ $5931,1.8762376216,-2.7052715628 \backslash \mathrm{H},-1.3066981847,0.7380920726,-3.663435$ $9427 \backslash \mathrm{H},-2.7028358981,2.3734674944,-2.6055665164 \backslash \mathrm{H},-0.5014371171,2.9898$ $345418,-3.3031593575 \backslash \mathrm{H}, 1.636654402,-0.4363206468,0.7544869612 \backslash 0,2.3722$ $158792,0.4923097594,2.3584694187 \backslash \mathrm{C}, 1.6036167223,1.0321582244,3.1320337$ $549 \backslash \mathrm{H}, 1.9798535583,1.5492623442,4.0342170494 \backslash \mathrm{H}, 0.5139813692,1.01803851$ $62,2.9552345236 \backslash \mathrm{Si},-1.4331352482,-1.4625940435,2.5524483704 \backslash \mathrm{H},-0.37215$ $72043,-2.468087195,2.2647625394 \backslash \mathrm{H},-1.233495415,-0.9825902656,3.9557284$ $921 \backslash \mathrm{H},-2.7447065393,-2.1769289397,2.5438773061 \backslash \mathrm{H},-2.1374454947,-2.1913$ $259517,-1.0418095376 \backslash \mathrm{H},-1.2057436639,-1.7676273005,-2.3277570515 \backslash \mathrm{H},-1$. $9177217223,0.5948444368,1.5638844295 \backslash \mathrm{H}, 1.2585705258,-1.7960524795,-0.1$ $022288713 \backslash \mathrm{H},-0.2359046865,2.139925155,-0.6542757699 \backslash \backslash$ Version=DEC $-\mathrm{AXP}-\mathrm{O}$ $\mathrm{SF} / 1-\mathrm{G} 03 \mathrm{RevB} .03 \backslash \mathrm{State}=1-\mathrm{A} \backslash \mathrm{HF}=-1453.7004844 \backslash \mathrm{RMSD}=5.922 \mathrm{e}-09 \backslash \mathrm{RMSF}=5.334 \mathrm{e}-$ $06 \backslash \mathrm{Dipole}=0.9363994,0.3586844,0.8877874 \backslash \mathrm{PG}=\mathrm{C} 01 \quad[\mathrm{X}(\mathrm{C} 1 \mathrm{H} 17 \mathrm{Al} 1 \mathrm{~N} 4 \mathrm{O} 1 \mathrm{Si} 3)] \backslash \backslash @$ 


\section{$\operatorname{TS}\left(3, \mathrm{CH}_{2} \mathrm{O}\right)$}

$1 \backslash 1 \backslash G I N C-S C 35 \backslash F T S \backslash R B 3 L Y P \backslash 6-31+G(d, p) \backslash C 1 H 19 A 11 N 401 S i 3 \backslash B U C 562 \backslash 03-M a r-200$ $5 \backslash 0 \backslash \backslash \#$ B3LYP $/ 6-31+G(D, P) \quad O P T=(T S, C A L C F C, N O E I G E N T E S T)$ MAXDISK=268435456

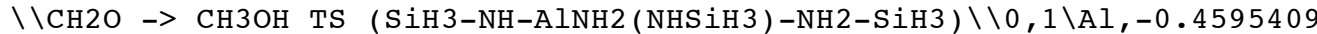
$913,-0.1492940268,-0.279629 \backslash \mathrm{N}, 0.9221380852,-1.4608749462,0.384699 \backslash \mathrm{N}, 0$. $6242629169,1.4246230364,-0.387334 \backslash N,-1.0010439557,-0.7592520584,-1.901$ $426 \backslash \mathrm{N},-1.7032609956,-0.0757410994,1.062871 \backslash \mathrm{Si}, 1.5187111553,-2.66210691$ $14,-0.82973 \backslash \mathrm{H}, 0.4107782085,-3.572998976,-1.179283 \backslash \mathrm{H}, 2.0308851116,-1.91$ $26838815,-1.993484 \backslash \mathrm{H}, 2.6199202006,-3.4385988471,-0.205091 \backslash \mathrm{Si}, 0.0342558$ $32,2.879314002,-1.210121 \backslash \mathrm{H},-1.3815581797,3.0790619194,-0.824909 \backslash \mathrm{H}, 0.83$ $73627632,4.0579790489,-0.790803 \backslash \mathrm{H}, 0.1358378396,2.7486980079,-2.687846 \backslash$ $\mathrm{H}, 1.7287790528,-0.9040518991,0.778217 \backslash 0,2.9019739847,0.2614441693,1.16$ $7897 \backslash \mathrm{C}, 2.6729469361,1.094818156,2.085226 \backslash \mathrm{H}, 3.2836638828,2.0084921916,2$ $.162946 \backslash \mathrm{H}, 1.3792958858,1.9574450805,1.759515 \backslash \mathrm{H}, 0.9808748944,1.81020005$ $72,0.940925 \backslash \mathrm{H}, 2.2196909549,0.7732971295,3.039364 \backslash \mathrm{Si},-3.392189973,-0.46$ $22471979,1.040795 \backslash \mathrm{H},-3.6447419186,-1.3950582127,-0.091432 \backslash \mathrm{H},-3.8188699$ $339,-1.1321332228,2.304466 \backslash \mathrm{H},-4.2975010416,0.7123677492,0.863472 \backslash \mathrm{H},-1$. $8523919249,-1.2876561081,-2.047863 \backslash \mathrm{H},-0.7105509794,-0.3522590415,-2.78$ $1394 \backslash \mathrm{H},-1.4108060235,0.4032959177,1.911609 \backslash \mathrm{H}, 0.487001113,-1.9369569716$ $, 1.177423 \backslash \mathrm{H}, 1.5773589305,1.191181092,-0.680092 \backslash \backslash$ Version=DEC-AXP-OSF $/ 1-$ G03RevB.03 $\backslash$ State $=1-A \backslash H F=-1454.8471027 \backslash R M S D=4.312 \mathrm{e}-09 \backslash R M S F=5.477 \mathrm{e}-06 \backslash \mathrm{Di}$ pole $=0.4044518,-0.2943454,0.0244071 \backslash$ Polar $=167.5371938,-7.5620803,159.8$ $539253,11.236057,-0.0315798,156.02304 \backslash \mathrm{PG}=\mathrm{C} 01 \quad[\mathrm{X}(\mathrm{C} 1 \mathrm{H} 19 \mathrm{~A} 11 \mathrm{~N} 4 \mathrm{O} 1 \mathrm{Si})] \backslash \backslash @$

\section{$3 \cdot \mathrm{CH}_{3} \mathrm{OH}$}

$1 \backslash 1 \backslash G I N C-S C 26 \backslash F O p t \backslash R B 3 L Y P \backslash 6-31+G(d, p) \backslash C 1 H 19 A 11 N 401 S i 3 \backslash B U C 562 \backslash 02-M a r-20$ $05 \backslash 0 \backslash \backslash \#$ B3LYP/6-31+G(D,P) OPT MAXDISK=268435456\\CH3OH SiH3-NH-AlNH2(N HSiH3 ) -NH2-SiH3 \\0, $1 \backslash \mathrm{Al},-0.2210943542,-0.6174965563,-0.64269281 \backslash \mathrm{N}, 1.65$ $21945642,-0.4879737286,-0.6619447175 \backslash \mathrm{N},-0.834924484,1.0636093261,0.321$ $6851427 \backslash \mathrm{N},-0.9843055199,-0.4142376082,-2.2878454623 \backslash \mathrm{N},-0.8506710653,-1$ $.9571274842,0.4531047855 \backslash \mathrm{Si}, 2.6914427678,-1.3042567175,0.4812687253 \backslash \mathrm{H}$, $4.119689475,-0.9892382527,0.1903967954 \backslash \mathrm{H}, 2.3683611905,-0.8202301587,1$. $8491481598 \backslash \mathrm{H}, 2.5692386555,-2.7923610131,0.4897179088 \backslash \mathrm{Si},-2.1521440473$, $1.9730637824,-0.5366344941 \backslash \mathrm{H},-3.2958047976,1.0690460807,-0.7586504063 \backslash$ $\mathrm{H},-2.5717944062,3.0720301936,0.375205223 \backslash \mathrm{H},-1.5975198168,2.5464983468$, $-1.7748073336 \backslash \mathrm{H}, 1.8996678266,1.3573620246,-0.3284204018 \backslash 0,1.6842148501$ $, 2.2936304625,-0.0867760486 \backslash \mathrm{C}, 2.8318285033,2.9742868374,0.4082435918 \backslash \mathrm{H}$ , $2.5235285411,3.9884105509,0.6748267423 \backslash \mathrm{H}, 3.2434678529,2.4871763361,1$. $3024856797 \backslash \mathrm{H},-0.0038549503,1.674507995,0.4210953601 \backslash \mathrm{H}, 3.6186530221,3.0$ $404505492,-0.3545594235 \backslash \mathrm{Si},-2.2271536559,-2.1140559431,1.4831850198 \backslash \mathrm{H}$, $-3.4467205761,-2.6949257757,0.8448643515 \backslash \mathrm{H},-1.9365833474,-2.9478290839$ $, 2.6840461682 \backslash \mathrm{H},-2.6151398792,-0.7481536084,1.9520711133 \backslash \mathrm{H},-1.67505606$ $83,-1.0705006221,-2.6319328932 \backslash \mathrm{H},-0.5581897962,0.0557819667,-3.0774251$ $039 \backslash \mathrm{H},-1.1288455932,0.807882424,1.2663904423 \backslash \mathrm{H},-0.3602963015,-2.840210$ $5464,0.324956995 \backslash \mathrm{H}, 2.010650386,-0.5594161409,-1.6141237619 \backslash \backslash$ Version=DE C-AXP-OSF $/ 1-G 03$ RevB. 03 \State $=1-\mathrm{A} \backslash \mathrm{HF}=-1454.9325802 \backslash \mathrm{RMSD}=8.626 \mathrm{e}-09 \backslash \mathrm{RMSF}=$ $2.382 e-06 \backslash \mathrm{Dipole}=0.1946215,0.7348021,-0.1788997 \backslash \mathrm{PG}=\mathrm{C} 01 \quad[\mathrm{X}(\mathrm{C} 1 \mathrm{H} 19 \mathrm{~A}] 1 \mathrm{~N} 401$ $\mathrm{Si3})] \backslash \backslash @$

4

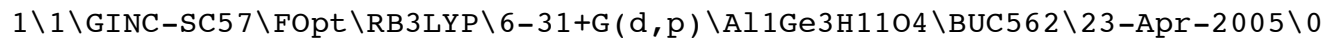
$\backslash \backslash \#$ B3LYP / 6-31+G(D,P) OPT MAXDISK $=671088640 \backslash \backslash \mathrm{GeH} 3-\mathrm{O}-\mathrm{AlOH}(\mathrm{OGeH} 3)-\mathrm{OH}-\mathrm{GeH}$ $3 \backslash \backslash 0,1 \backslash \mathrm{Al},-0.3749169941,-0.0639730348,0.339184 \backslash 0,-1.7955119817,-0.1969$ $131666,-0.927526 \backslash 0,0.4160618736,1.3617170386,-0.238759 \backslash 0,-1.5948400078$ $, 0.083980852,1.601987 \backslash 0,0.5626871402,-1.5101339478,0.426653 \backslash \mathrm{Ge},-3.5034$ $38998,-0.0215683252,-0.171336 \backslash \mathrm{H},-3.6714681228,1.3225816592,0.525018 \backslash \mathrm{H}$, $-4.3088819972,-0.0300123999,-1.485765 \backslash \mathrm{H},-3.7867978798,-1.2954973515,0$. $613853 \backslash \mathrm{Ge}, 2.0463868106,2.0407361899,-0.038109 \backslash \mathrm{H}, 2.7193208634,1.4722652$ $524,1.227393 \backslash \mathrm{H}, 2.8894398437,1.6839242682,-1.2789 \backslash \mathrm{H}, 1.909299669,3.56608$ $81772,0.084456 \backslash \mathrm{H},-1.6878710225,0.2429538433,-1.781816 \backslash \mathrm{Ge}, 2.1960771799$, $-1.9381817962,-0.127869 \backslash \mathrm{H}, 2.4990231191,-1.2829407681,-1.490644 \backslash \mathrm{H}, 3.226$ $4681358,-1.4634937005,0.917125 \backslash \mathrm{H}, 2.2522323218,-3.467022791,-0.263986 \backslash \mathrm{H}$ $,-1.5227929612,-0.4179681413,2.419074 \backslash \backslash$ Version=Al64T-G03RevC.02 $\backslash$ State $=$ $1-\mathrm{A} \backslash \mathrm{HF}=-6775.2498333 \backslash \mathrm{RMSD}=2.548 \mathrm{e}-09 \backslash \mathrm{RMSF}=7.002 \mathrm{e}-06 \backslash \mathrm{Dipole}=-0.9845022,0$ $.0011847,-0.4469377 \backslash \mathrm{PG}=\mathrm{C} 01 \quad[\mathrm{X}(\mathrm{A} 11 \mathrm{Ge} 3 \mathrm{H} 11 \mathrm{O} 4)] \backslash \backslash @$ 
$4 \cdot \mathrm{CO}_{2}$

$1 \backslash 1 \backslash$ GINC-SC60 $\backslash$ FOpt $\backslash R B 3 L Y P \backslash 6-31+G(d, p) \backslash C 1 H 11 A 11 \mathrm{Ge} 306 \backslash B U C 562 \backslash 23-A p r-2005$ $\backslash 0 \backslash \backslash \#$ B3LYP/6-31+G(D,P) OPT MAXDISK=671088640\\CO2 - GeH3-O-Al(OH) OGeH $3-\mathrm{OH}-\mathrm{GeH} 3 \backslash \backslash 0,1 \backslash \mathrm{Al},-0.941561333,0.1435422502,0.4001102898 \backslash 0,-0.70112987$ $59,1.7244427319,-0.649784648 \backslash 0,0.6905319289,-0.4563447417,0.3364822939$ $\backslash 0,-1.327430882,1.0392200045,1.8552083282 \backslash 0,-2.1503571709,-0.852744452$ $8,-0.3196742447 \backslash \mathrm{Ge},-0.1384598052,3.1360763976,0.4380881359 \backslash \mathrm{H}, 0.8676948$ $457,2.6266199185,1.460489002 \backslash \mathrm{H}, 0.5460650491,4.0303110837,-0.6074893275$ $\backslash \mathrm{H},-1.407486608,3.7828956395,0.9760378347 \backslash \mathrm{Ge}, 1.6533679737,-1.284582006$ , $1.5846416937 \backslash \mathrm{H}, 0.85330368,-1.3907451742,2.8932013306 \backslash \mathrm{H}, 2.0139603663,-$ $2.6857543224,1.0634388848 \backslash \mathrm{H}, 2.9306454606,-0.453624924,1.8127949216 \backslash \mathrm{H},-$ $0.0854343844,1.588790925,-1.3917254037 \backslash 0,1.5088540984,0.7632627604,-2$. $291913973 \backslash \mathrm{C}, 2.2608605917,-0.0208360282,-1.8441625906 \backslash 0,3.0666567964,-0$ $.7734436046,-1.4685304863 \backslash \mathrm{Ge},-1.7811636306,-2.2788896607,-1.3203241502$ $\backslash \mathrm{H},-0.6678317747,-1.9518429498,-2.3366581462 \backslash \mathrm{H},-1.3141386532,-3.439001$ $9014,-0.4174810581 \backslash \mathrm{H},-3.0654561655,-2.671146676,-2.0624746797 \backslash \mathrm{H},-2.173$ $0124088,0.94397233,2.302128518 \backslash \backslash$ Version=Al64T-G03RevC.02 $\backslash$ State $=1-\mathrm{A} \backslash \mathrm{HF}=$ $-6963.8554974 \backslash \mathrm{RMSD}=8.185 \mathrm{e}-09 \backslash \mathrm{RMSF}=1.638 \mathrm{e}-05 \backslash \mathrm{Dipole}=0.4442909,1.0758836$ ,$-0.1214237 \backslash \mathrm{PG}=\mathrm{C} 01 \quad[\mathrm{X}(\mathrm{C} 1 \mathrm{H} 11 \mathrm{Al} 1 \mathrm{Ge} 3 \mathrm{O} 6)] \backslash \backslash @$

\section{$\operatorname{TS}\left(4, \mathrm{CO}_{2}\right)$}

$1 \backslash 1 \backslash G I N C-S C 67 \backslash F T S \backslash R B 3 L Y P \backslash 6-31+G(d, p) \backslash C 1 H 13 A l 1 G e 306 \backslash B U C 562 \backslash 24-A p r-2005 \backslash$ $0 \backslash \backslash \#$ B3LYP/6-31+G (D,P) OPT=(TS,CALCFC, NOEIGENTEST) MAXDISK=671088640 \ $\mathrm{CO} 2->\mathrm{HCO} 2 \mathrm{H}$ TS $(\mathrm{GeH} 3-\mathrm{O}-\mathrm{AlOH}(\mathrm{OGeH} 3)-\mathrm{OH}-\mathrm{GeH} 3) \backslash \backslash 0,1 \backslash \mathrm{Al},-0.798532011,-0.4$ $53268704,-0.2068170613 \backslash 0,0.5437816472,-1.7359557824,-0.1083692935 \backslash 0,0$. $1709619818,1.0612502393,-0.1234726829 \backslash 0,-1.2138532717,-0.668800399,-1$. $8907251867 \backslash 0,-1.9595313103,-0.6077342078,1.0421841169 \backslash \mathrm{Ge}, 1.2815164192$, $-2.1872968767,-1.7549046477 \backslash \mathrm{H}, 1.6010959991,-0.8963787394,-2.5004849766$ $\backslash \mathrm{H}, 2.5509255967,-2.9310468222,-1.3361761386 \backslash \mathrm{H}, 0.2668469022,-3.09962657$ $73,-2.4306470805 \backslash \mathrm{Ge}, 0.1073245834,2.2616042299,-1.4903083967 \backslash \mathrm{H}, 0.824264$ $3858,3.5033949489,-0.9566489064 \backslash \mathrm{H}, 0.8352603079,1.6763445853,-2.7032777$ $597 \backslash \mathrm{H},-1.3700815103,2.5380731399,-1.7778394384 \backslash \mathrm{H}, 1.257231962,-1.557593$ $4894,0.5715004581 \backslash 0,2.4225348608,-1.096991377,1.5922755008 \backslash \mathrm{C}, 2.7578048$ $182,0.0051302025,1.983210196 \backslash 0,3.462122784,0.6533301259,2.6877157081 \backslash \mathrm{H}$ $, 1.9211869416,1.0100520863,1.256219713 \backslash \mathrm{H}, 1.2039069408,1.0848938734,0.6$ $809903905 \backslash \mathrm{Ge},-2.4172603811,0.6057675955,2.2689102765 \backslash \mathrm{H},-1.1660583405,1$ $.0673033147,3.0363036157 \backslash \mathrm{H},-3.0715190716,1.8136312427,1.5685489441 \backslash \mathrm{H},-$ $3.4180774649,-0.062727676,3.2174177956 \backslash \mathrm{H},-2.0996088376,-0.8877951007,-$ $2.1917227251 \backslash \backslash$ Version $=A 164 \mathrm{~T}-\mathrm{G} 03$ RevC.02 $\backslash$ State $=1-\mathrm{A} \backslash \mathrm{HF}=-6964.9921585 \backslash \mathrm{RMSD}$ $=7.085 e-09 \backslash \mathrm{RMSF}=3.688 \mathrm{e}-06 \backslash \mathrm{Dipole}=-0.5746613,-0.0212536,-1.871306 \backslash \mathrm{PG}=\mathrm{C} 0$ $1[\mathrm{X}(\mathrm{C} 1 \mathrm{H} 13 \mathrm{Al} 1 \mathrm{Ge} 3 \mathrm{O} 6)] \backslash \backslash \mathrm{Q}$

\section{$4 \cdot \mathrm{HCO}_{2} \mathrm{H}$}

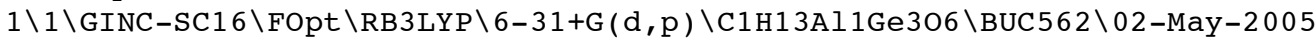
$\backslash 0 \backslash \backslash \#$ B3LYP $/ 6-31+G(D, P)$ OPT MAXDISK=671088640 \لHCO2H - GeH3-O-AlOH $(\mathrm{OGe}$ H3 ) $-\mathrm{OH}-\mathrm{GeH} 3 \backslash \backslash 0,1 \backslash \mathrm{Al},-0.135811936,-0.511897017,-0.83151 \backslash 0,-0.2119199618$ $,-0.3053920265,0.928895 \backslash 0,1.2293659075,0.7392421538,-1.241307 \backslash 0,0.8040$ $312422,-1.9356938994,-1.216953 \backslash 0,-1.5890789799,-0.1605431988,-1.675074$ $\backslash \mathrm{Ge},-0.1258817833,-1.7321930158,2.021971 \backslash \mathrm{H}, 1.3340532737,-2.1876918331$, $2.148869 \backslash \mathrm{H},-0.6612768422,-1.2614880827,3.379418 \backslash \mathrm{H},-1.0060116437,-2.847$ $6821259,1.443511 \backslash \mathrm{Ge}, 2.9256710029,-0.0230106339,-1.037765 \backslash \mathrm{H}, 3.189074098$ $8,-0.789991601,-2.325604 \backslash \mathrm{H}, 3.7963288458,1.232065475,-0.9201 \backslash \mathrm{H}, 2.940139$ $1049,-0.8382256321,0.249074 \backslash \mathrm{H}, 0.2929728544,1.1638780367,1.244276 \backslash 0,0.7$ $241227413,2.0675920906,1.075027 \backslash \mathrm{C}, 0.1640846146,3.0805080205,1.760701 \backslash 0$ $, 0.4999144708,4.2296350625,1.628472 \backslash \mathrm{H},-0.6271323445,2.7530799215,2.457$ $716 \backslash \mathrm{H}, 1.1691458048,1.5596881463,-0.705709 \backslash \mathrm{Ge},-3.1462920381,0.304152606$ $3,-0.940384 \backslash \mathrm{H},-2.9510241921,1.5352436308,-0.034175 \backslash \mathrm{H},-4.113910079,0.63$ $15914853,-2.082733 \backslash \mathrm{H},-3.6908058914,-0.8680734618,-0.099536 \backslash \mathrm{H}, 0.5460693$ $198,-2.5558849317,-1.904345 \backslash \backslash$ Version=Al $64 \mathrm{~T}-\mathrm{G} 03$ RevC.02 $\backslash$ State $=1-\mathrm{A} \backslash \mathrm{HF}=-69$ $65.0518416 \backslash \mathrm{RMSD}=3.859 \mathrm{e}-09 \backslash \mathrm{RMSF}=1.589 \mathrm{e}-05 \backslash \mathrm{Dipole}=0.542214,-1.4605035,0$. $1017687 \backslash \mathrm{PG}=\mathrm{C} 01 \quad[\mathrm{X}(\mathrm{C} 1 \mathrm{H} 13 \mathrm{Al} 1 \mathrm{Ge} 3 \mathrm{O} 6)] \backslash \backslash \mathrm{Q}$ 


\section{$\operatorname{TS}\left(4, \mathrm{HCO}_{2} \mathrm{H}\right)$}

$1 \backslash 1 \backslash G I N C-S C 26 \backslash F T S \backslash R B 3 L Y P \backslash 6-31+G(d, p) \backslash C 1 H 15 A l 1 G e 306 \backslash B U C 562 \backslash 25-A p r-2005 \backslash$ $0 \backslash \backslash \#$ B3LYP $/ 6-31+G(D, P) \quad O P T=(T S, C A L C F C, N O E I G E N T E S T) \quad M A X D I S K=671088640 \backslash \backslash$ $\mathrm{HCO} 2 \mathrm{H} \rightarrow \mathrm{CH} 2(\mathrm{OH}) 2$ TS $(\mathrm{GeH} 3-\mathrm{O}-\mathrm{AlOH}(\mathrm{OGeH} 3)-\mathrm{OH}-\mathrm{GeH} 3) \backslash \backslash 0,1 \backslash \mathrm{Al},-0.235538164$ $2,-0.1103690326,-0.7703273129 \backslash 0,-0.1001736719,-0.2660382978,1.04267607$ $79 \backslash 0,1.3166400055,0.6988685187,-1.2074577523 \backslash 0,-0.1848486469,-1.803360$ $3216,-1.2017531004 \backslash 0,-1.5689154182,0.8952529598,-1.201396556 \backslash \mathrm{Ge},-0.144$ $7090152,-1.9066652204,1.836709334 \backslash \mathrm{H},-1.4650574918,-2.5612789704,1.4359$ $856765 \backslash \mathrm{H}, 1.0844339412,-2.7057706906,1.4060987577 \backslash \mathrm{H},-0.1023778557,-1.58$ $37400834,3.3335767449 \backslash \mathrm{Ge}, 2.7773506902,-0.3753470796,-1.370962834 \backslash \mathrm{H}, 2.8$ $50269333,-1.3123525375,-0.1624728075 \backslash \mathrm{H}, 2.6336944112,-1.1392287298,-2.6$ $891537332 \backslash \mathrm{H}, 3.9714002121,0.5829675601,-1.402413941 \backslash \mathrm{H}, 0.3972085572,0.60$ $19534576,1.5611851815 \backslash 0,1.0159197713,1.6396393101,2.0112587353 \backslash \mathrm{C}, 0.837$ $9402075,2.7132414198,1.3384517207 \backslash 0,1.433324132,3.8490158184,1.7806697$ $031 \backslash \mathrm{H}, 1.5311293449,2.5485688982,0.0004964646 \backslash \mathrm{H}, 1.4930026996,1.80185889$ $04,-0.5667508546 \backslash \mathrm{H},-0.124341214,2.9400633801,0.8705061691 \backslash \mathrm{H}, 2.14703140$ $4,3.5842510288,2.3842368026 \backslash \mathrm{Ge},-3.2547677742,0.5090346907,-0.778469343$ $4 \backslash \mathrm{H},-4.1755543971,1.3240070503,-1.6917271219 \backslash \mathrm{H},-3.5108227212,0.8746619$ $508,0.6973659707 \backslash \mathrm{H},-3.5153631474,-0.9983111791,-0.9778652091 \backslash \mathrm{H},-0.5678$ $323862,-2.1740415256,-2.0003692261 \backslash \backslash$ Version=Al64T-G03RevC.02 $\backslash$ State $=1-A$ $\backslash \mathrm{HF}=-6966.1815069 \backslash \mathrm{RMSD}=5.327 \mathrm{e}-09 \backslash \mathrm{RMSF}=1.982 \mathrm{e}-06 \backslash \mathrm{Dipole}=0.572256,-0.809$ $4804,0.2926476 \backslash \mathrm{PG}=\mathrm{C} 01 \quad[\mathrm{X}(\mathrm{C} 1 \mathrm{H} 15 \mathrm{Al} 1 \mathrm{Ge} 306)] \backslash \backslash @$

\section{$4 \cdot \mathrm{CH}_{2}(\mathrm{OH})_{2}$}

$1 \backslash 1 \backslash \mathrm{GINC}-\mathrm{SC} 76 \backslash \mathrm{FOpt} \backslash \mathrm{RB} 3 \mathrm{LYP} \backslash 6-31+\mathrm{G}(\mathrm{d}, \mathrm{p}) \backslash \mathrm{C} 1 \mathrm{H} 15 \mathrm{Al} 1 \mathrm{Ge} 306 \backslash \mathrm{BUC} 562 \backslash 24-\mathrm{Apr}-2005$ $\backslash 0 \backslash \backslash \#$ B3LYP $/ 6-31+G(D, P)$ OPT MAXDISK=671088640 \لCH2(OH) 2 - GeH3-O-AlOH ( $\mathrm{OGeH} 3$ ) $-\mathrm{OH}-\mathrm{GeH} 3 \backslash \backslash 0,1 \backslash \mathrm{Al}, 0.3835426971,-0.801346145,0.586538 \backslash 0,-0.4889937$ $283,0.7188631848,0.737563 \backslash 0,1.7292929245,-0.1996616537,-0.609435 \backslash 0,1.5$ $462565406,-1.2153555845,1.824835 \backslash 0,-0.5401848,-2.1162047958,-0.037861 \backslash$ $\mathrm{Ge},-2.1328475604,1.1629738062,1.284813 \backslash \mathrm{H},-2.7101449952,0.0127700245,2$. $126322 \backslash \mathrm{H},-1.9956830785,2.4376927544,2.132575 \backslash \mathrm{H},-3.0039124628,1.4210331$ $355,0.045254 \backslash \mathrm{Ge}, 3.4688808909,-0.2885443113,0.038235 \backslash \mathrm{H}, 3.804784331,-1.7$ $697394383,0.158567 \backslash \mathrm{H}, 4.2370591345,0.3558003983,-1.126263 \backslash \mathrm{H}, 3.600301207$ $9,0.550026639,1.305532 \backslash \mathrm{H}, 0.2328876993,1.849832912,-0.225527 \backslash 0,0.832904$ $8614,2.2788616852,-0.904253 \backslash \mathrm{C}, 0.0266590971,2.9022719899,-1.885389 \backslash 0,-0$ $.9143875739,3.7727333457,-1.303183 \backslash \mathrm{H}, 0.7142612952,3.42620573,-2.559676$ $\backslash \mathrm{H}, 1.5403742667,0.7055964177,-0.976388 \backslash \mathrm{H},-0.5671211789,2.1720622144,-2$ $.446553 \backslash \mathrm{H},-0.4379303222,4.4385841655,-0.786149 \backslash \mathrm{Ge},-2.0232508041,-2.127$ $1482352,-1.014179 \backslash \mathrm{H},-3.2375058767,-2.3191327762,-0.083397 \backslash \mathrm{H},-2.1816923$ $046,-0.8057621753,-1.794936 \backslash \mathrm{H},-1.9276452476,-3.3003792713,-2.002355 \backslash \mathrm{H}$, $1.4578222449,-1.9976105511,2.375196 \backslash \backslash$ Version=Al64T-G03RevC.02\State=1$\mathrm{A} \backslash \mathrm{HF}=-6966.2486523 \backslash \mathrm{RMSD}=9.550 \mathrm{e}-09 \backslash \mathrm{RMSF}=7.536 \mathrm{e}-06 \backslash \mathrm{Dipole}=0.7046894,0.55$ $37652,-0.1927265 \backslash \mathrm{PG}=\mathrm{C} 01 \quad[\mathrm{X}(\mathrm{C} 1 \mathrm{H} 15 \mathrm{Al} 1 \mathrm{Ge} 306)] \backslash \backslash @$

\section{$4 \cdot \mathrm{CH}_{2} \mathrm{O}$}

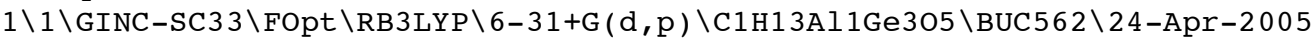
$\backslash 0 \backslash \backslash \#$ B3LYP $/ 6-31+G(D, P)$ OPT MAXDISK $=671088640 \backslash \backslash \mathrm{CH} 2 \mathrm{O}$ GeH3-O-AlOH $(\mathrm{OGeH} 3)$ $-\mathrm{OH}-\mathrm{GeH} 3 \backslash \backslash 0,1 \backslash \mathrm{Al}, 0.3594216416,-0.244220968,-0.2264065902 \backslash 0,0.059019405$ $1,1.4567735364,0.5400677199 \backslash 0,-1.1042408342,-1.100887886,0.2088537591 \backslash$ $0,0.2051899388,0.3607628642,-1.8788728871 \backslash 0,1.8424328783,-0.870743661$, $0.3110574085 \backslash \mathrm{Ge},-0.7133859508,2.6637737626,-0.6389762752 \backslash \mathrm{H}, 0.393709412$ $9,3.1675447122,-1.5560555146 \backslash \mathrm{H},-1.9238632489,2.0333391596,-1.311642042$ $\backslash \mathrm{H},-1.1533218364,3.7654331896,0.3376373964 \backslash \mathrm{Ge},-2.3855683673,-1.3644281$ $216,-1.0062877141 \backslash \mathrm{H},-1.826236634,-2.036563654,-2.2702830961 \backslash \mathrm{H},-3.42333$ $28203,-2.2875343029,-0.3472502638 \backslash \mathrm{H},-3.068260468,-0.0327822822,-1.3700$ $029714 \backslash \mathrm{H},-0.3801093753,1.4614082072,1.4310167547 \backslash \mathrm{O},-1.2080285117,1.217$ $8772501,2.9080804085 \backslash \mathrm{C},-1.8272504071,0.1689438159,3.0136972028 \backslash \mathrm{H},-2.34$ $94977345,-0.067203094,3.9575247801 \backslash \mathrm{H},-1.8676625072,-0.5512776829,2.178$ $3940729 \backslash \mathrm{Ge}, 3.4410476725,-1.5166640138,0.6290266277 \backslash \mathrm{H}, 4.287758316,-0.43$ $28593823,1.3224154515 \backslash \mathrm{H}, 3.3098390208,-2.7478602103,1.5438330426 \backslash \mathrm{H}, 4.10$ $04802235,-1.921212988,-0.7038443527 \backslash \mathrm{H}, 0.8895484025,0.2547091174,-2.544$ $5365025 \backslash \backslash$ Version=Al64 T-G03RevC. 02 \State $=1-\mathrm{A} \backslash \mathrm{HF}=-6889.7832751 \backslash \mathrm{RMSD}=3.42$ $1 e-09 \backslash \mathrm{RMSF}=5.592 \mathrm{e}-05 \backslash \mathrm{Dipole}=-0.6358591,0.5595439,0.3233636 \backslash \mathrm{PG}=\mathrm{C} 01 \quad[\mathrm{X}(\mathrm{C}$ $1 \mathrm{H} 13 \mathrm{Al} 1 \mathrm{Ge} 305) \mathrm{J} \backslash \backslash \mathrm{Q}$ 


\section{$\operatorname{TS}\left(4, \mathrm{CH}_{2} \mathrm{O}\right)$}

$1 \backslash 1 \backslash G I N C-S C 125 \backslash F T S \backslash R B 3 L Y P \backslash 6-31+G(d, p) \backslash C 1 H 15 A 11 G e 305 \backslash B U C 562 \backslash 23-A p r-2005$ $\backslash 0 \backslash \backslash \#$ B3LYP /6-31+G(D,P) OPT=(TS,CALCFC, NOEIGENTEST ) MAXDISK=671088640 $\backslash \mathrm{CH} 2 \mathrm{O} \rightarrow \mathrm{CH} 3 \mathrm{OH}$ TS (GeH3-O-AlOH $(\mathrm{OGeH} 3)-\mathrm{OH}-\mathrm{GeH} 3) \backslash \backslash 0,1 \backslash \mathrm{Al}, 0.1995051523,-0$ $.6399398434,-0.1843486215 \backslash 0,-0.1602181332,1.1032005606,0.2213246045 \backslash 0$, $-1.1317349922,-1.5332062575,0.6200517765 \backslash 0,0.0291510582,-0.5511475182$, $-1.9208219132 \backslash 0,1.692691243,-1.1154862891,0.551980436 \backslash \mathrm{Ge},-0.3746722774$ $, 2.3402845129,-1.0972318399 \backslash \mathrm{H}, 0.9061932956,2.3442690516,-1.9299021952 \backslash$ $\mathrm{H},-1.623103513,1.9915453219,-1.9062864524 \backslash \mathrm{H},-0.5546494734,3.6567694619$ $,-0.3340752314 \backslash \mathrm{Ge},-2.7592802397,-1.4958002868,-0.173987652 \backslash \mathrm{H},-2.722435$ $6292,-2.4702278067,-1.3567879175 \backslash \mathrm{H},-3.7533462469,-1.9587534103,0.89591$ $72261 \backslash \mathrm{H},-3.07331095,-0.0671525585,-0.632100953 \backslash \mathrm{H},-0.464231324,1.266849$ $8411,1.3044087424 \backslash 0,-0.8203614052,1.2182478161,2.5323037252 \backslash \mathrm{C},-0.29832$ $07574,0.2462335688,3.1633882627 \backslash \mathrm{H},-0.5563808351,0.1217197271,4.2208618$ $121 \backslash \mathrm{H},-1.0302281505,-1.111880027,2.8503745913 \backslash \mathrm{H},-1.0963645887,-1.35071$ $19754,2.0021133296 \backslash \mathrm{H}, 0.6679788615,-0.1640768354,2.8453916716 \backslash \mathrm{Ge}, 3.2637$ $741127,-0.4223483804,0.0859614006 \backslash \mathrm{H}, 3.449693226,0.9384685071,0.7895952$ $387 \backslash \mathrm{H}, 4.3651159212,-1.392273589,0.527772955 \backslash \mathrm{H}, 3.3236493385,-0.21425104$ $77,-1.4402082636 \backslash \mathrm{H}, 0.3272644104,-1.229691666,-2.5313221608 \backslash \backslash$ Version $=\mathrm{Al}$ $64 \mathrm{~T}-\mathrm{G} 03 \mathrm{RevC} .02 \backslash$ State $=1-\mathrm{A} \backslash \mathrm{HF}=-6890.935611 \backslash \mathrm{RMSD}=4.978 \mathrm{e}-09 \backslash \mathrm{RMSF}=8.628 \mathrm{e}-06$ $\backslash$ Dipole $=-0.3000895,0.1585126,-0.0733153 \backslash P G=C 01 \quad[X(C 1 H 15 A 11 G e 305)] \backslash \backslash @$

\section{$4 \cdot \mathrm{CH}_{3} \mathrm{OH}$}

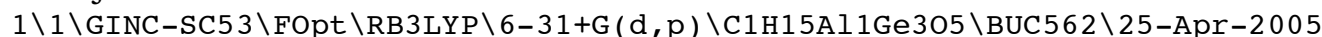
$\backslash 0 \backslash \backslash \#$ B3LYP /6-31+G(D,P) OPT MAXDISK=671088640 \CH3OH GeH3-O-AlOH (OGeH3 ) $-\mathrm{OH}-\mathrm{GeH} 3 \backslash \backslash 0,1 \backslash \mathrm{Al}, 0.0811210115,-0.729993277,-0.3932369519 \backslash 0,0.37739026$ $87,0.9773221619,-0.0437658478 \backslash 0,-1.5967106117,-0.9222586266,0.44948445$ $63 \backslash 0,-0.4742390411,-0.9611001752,-2.0373566412 \backslash 0,1.2497245236,-1.80629$ $49498,0.2782119073 \backslash \mathrm{Ge}, 0.8336228393,2.1518187906,-1.3154956654 \backslash \mathrm{H},-0.406$ $197936,2.5343217628,-2.1387959626 \backslash \mathrm{H}, 1.3879860579,3.3873790272,-0.59310$ $66465 \backslash \mathrm{H}, 1.9102217261,1.5315728253,-2.2200695418 \backslash \mathrm{Ge},-2.9899308462,-0.52$ $05559198,-0.711637726 \backslash \mathrm{H},-3.2170626363,-1.7761599634,-1.5443521508 \backslash \mathrm{H},-4$ $.1471089179,-0.2707332652,0.263274564 \backslash \mathrm{H},-2.6466720371,0.7414371172,-1$. $4948268088 \backslash \mathrm{H},-0.5657965712,1.317738246,1.4091709683 \backslash 0,-1.2485803569,1$. $0633138338,2.0792548014 \backslash \mathrm{C},-0.6890962525,1.0832888707,3.3952258319 \backslash \mathrm{H},-1$ $.4447124237,0.690820214,4.0791848004 \backslash \mathrm{H}, 0.2106948284,0.4597356927,3.459$ $0796095 \backslash \mathrm{H},-1.6206814929,-0.2926327778,1.2355388873 \backslash \mathrm{H},-0.4400907041,2.1$ $081917196,3.6928766723 \backslash \mathrm{Ge}, 2.7269824055,-1.2919794279,1.1293858125 \backslash \mathrm{H}, 2$. $3691430883,-0.3978844396,2.333975349 \backslash \mathrm{H}, 3.4411853727,-2.5579680066,1.61$ $86059658 \backslash \mathrm{H}, 3.6458370635,-0.5054665395,0.1725905661 \backslash \mathrm{H},-0.1190000656,-1$. $6451003613,-2.6111277712 \backslash \backslash$ Version=Al64T-G03RevC. 02 $\backslash$ State $=1-A \backslash H F=-6891$. $0179504 \backslash \mathrm{RMSD}=6.113 \mathrm{e}-09 \backslash \mathrm{RMSF}=7.276 \mathrm{e}-06 \backslash \mathrm{Dipole}=-0.6057328,0.4306855,0.26$ $16887 \backslash \mathrm{PG}=\mathrm{C} 01 \quad[\mathrm{X}(\mathrm{C} 1 \mathrm{H} 15 \mathrm{Al} 1 \mathrm{Ge} 305)] \backslash \backslash @$

\section{5}

$1 \backslash 1 \backslash G I N C-L C 80 \backslash F O p t \backslash R B 3 L Y P \backslash 6-31+G(d, p) \backslash A l 1 G e 3 H 14 N 4 N a 1 \backslash B U C 562 \backslash 06-J u 1-200$

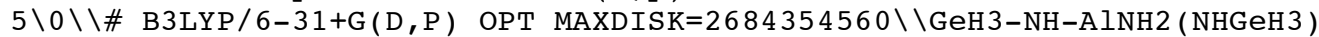
$-\mathrm{NHNa}-\mathrm{GeH} 3 \backslash \backslash 0,1 \backslash \mathrm{Ge},-3.3945145398,-1.6725684934,0.0001350961 \backslash \mathrm{N},-1.61540$ $96744,-1.4061347177,-0.0001473321 \backslash \mathrm{Al},-0.3365883277,-0.0953426967,-0.00$ $03743977 \backslash \mathrm{Na}, 2.4249354037,-1.1941681602,0.0014473425 \backslash \mathrm{N}, 0.8493320192,-0$. $4084859792,1.4759909507 \backslash \mathrm{Ge}, 1.4837896522,1.0652383457,2.3747679113 \backslash \mathrm{N},-0$ $.739327217,1.6962942548,-0.0016117495 \backslash \mathrm{N}, 0.850565051,-0.4103083447,-1.4$ $753472422 \backslash \mathrm{H}, 0.4125488561,2.0767341036,2.8254730751 \backslash \mathrm{H}, 2.238501376,0.596$ $2327794,3.6393295204 \backslash \mathrm{H}, 2.503804593,1.7737436493,1.4618608599 \backslash \mathrm{H},-4.1041$ $6728,-0.3079277588,-0.0013345155 \backslash \mathrm{H},-3.865114665,-2.480589074,-1.235669$ $6733 \backslash \mathrm{H},-3.8653474262,-2.4779151653,1.2376172205 \backslash \mathrm{Ge}, 1.4859598892,1.0625$ $689303,-2.3748950528 \backslash \mathrm{H},-1.141237037,2.1449958716,0.8151378343 \backslash \mathrm{H}, 2.5048$ $373176,1.7720648329,-1.4614941752 \backslash \mathrm{H}, 0.4151853109,2.0734820222,-2.82798$ $32778 \backslash \mathrm{H}, 2.242250813,0.5922719442,-3.6380385361 \backslash \mathrm{H},-1.1704462214,-2.3265$ $364486,-0.00019832 \backslash \mathrm{H}, 0.4249295884,-1.0373772304,-2.1580016709 \backslash \mathrm{H}, 0.4231$ $031904,-1.0349558726,2.1588265445 \backslash \mathrm{H},-1.1411308977,2.1438836331,-0.8190$ $254176 \backslash \backslash$ Version=IA32L-G03RevC .02 \State $=1-A \backslash H F=-6857.4497059 \backslash$ RMSD $=4.704$ e-09\RMSF $=6.650 e-06 \backslash \mathrm{Dipole}=2.3747474,-1.4376365,0.0014894 \backslash \mathrm{PG}=\mathrm{C} 01 \quad[\mathrm{X}(\mathrm{A}]$ $1 \mathrm{Ge} 3 \mathrm{H} 14 \mathrm{~N} 4 \mathrm{Na} 1)] \backslash \backslash \mathrm{Q}$ 
$\mathbf{5} \cdot \mathrm{CO}_{2}$

$1 \backslash 1 \backslash$ GINC-AC50 $\backslash$ FOpt $\backslash R B 3 L Y P \backslash 6-31+G(d, p) \backslash C 1 \mathrm{H} 14 \mathrm{Al} 1 \mathrm{Ge} 3 \mathrm{~N} 4 \mathrm{Na} 102 \backslash \mathrm{BUC5} 62 \backslash 12-\mathrm{Ju} 1$ $-2005 \backslash 0 \backslash \backslash \#$ B3LYP/6-31+G(D,P) OPT MAXDISK=20000MB \\CO2 - GeH3-NH-AlNH2(

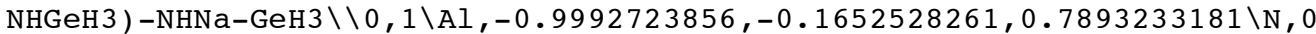
$.3701152795,-1.4369678548,0.469637868 \backslash \mathrm{N},-2.054744866,-0.8743952997,-0$. $6207027226 \backslash \mathrm{N},-1.466799582,0.0428845811,2.5321687595 \backslash \mathrm{N},-0.436443548,1.4$ $057425122,-0.0887503381 \backslash \mathrm{Ge}, 1.9492325791,-1.6745994276,1.3258056458 \backslash \mathrm{H}, 1$ $.8546598055,-2.2492794607,2.7548164053 \backslash \mathrm{H}, 2.8232171441,-2.6592510983,0$. $5031244616 \backslash \mathrm{H}, 2.6926299686,-0.3280388869,1.4046318693 \backslash \mathrm{Ge},-3.6578893834$, $-0.4044462499,-1.314040912 \backslash \mathrm{H},-3.9481592894,-1.21796337,-2.5964127376 \backslash \mathrm{H}$ $,-4.8666786785,-0.5914146403,-0.3680245962 \backslash \mathrm{H},-3.5889831056,1.089578499$ $2,-1.7030592461 \backslash \mathrm{Na}, 0.1413098056,-0.3260462811,-1.6669711133 \backslash 0,2.560905$ $7725,-0.2959264834,-2.0391939195 \backslash \mathrm{C}, 3.5860734782,-0.443943288,-1.484519$ $6602 \backslash 0,4.6128413853,-0.5863635702,-0.9589196299 \backslash \mathrm{Ge}, 0.6610408769,2.7166$ $08163,0.5357557531 \backslash \mathrm{H},-1.5716268345,-0.7219076478,3.1874053676 \backslash \mathrm{H}, 1.9676$ $011173,2.074663584,1.0306416784 \backslash \mathrm{H}, 1.0052673085,3.6940844783,-0.6135301$ $633 \backslash \mathrm{H}, 0.0622013535,3.5518604104,1.6904950414 \backslash \mathrm{H},-2.0560258327,-1.894106$ $4972,-0.555225917 \backslash \mathrm{H},-1.2240676782,1.8455889157,-0.5677096484 \backslash \mathrm{H},-0.0439$ $574218,-2.3606145851,0.3333616413 \backslash \mathrm{H},-1.9375441427,0.8607291773,2.89986$ $07582 \backslash \backslash$ Version=IA64L-G03RevC. 02 \State $=1-A \backslash \mathrm{HF}=-7046.06447 \backslash \mathrm{RMSD}=6.441 \mathrm{e}-0$ $9 \backslash \mathrm{RMSF}=9.104 \mathrm{e}-06 \backslash \mathrm{Dipole}=0.5517535,-0.6118086,-1.8064628 \backslash \mathrm{PG}=\mathrm{C} 01 \quad[\mathrm{X}(\mathrm{C} 1 \mathrm{H} 1$ $4 \mathrm{Al} 1 \mathrm{Ge} 3 \mathrm{~N} 4 \mathrm{Na} 1 \mathrm{O} 2$ ) $] \backslash \backslash$ @

\section{$\operatorname{TS}\left(5, \mathrm{CO}_{2}\right)$}

$1 \backslash 1 \backslash$ GINC-AC9 \FTS $\backslash R B 3 L Y P \backslash 6-31+G(d, p) \backslash C 1 H 16 A 11 \mathrm{Ge} 3 \mathrm{~N} 4 \mathrm{Na} 102 \backslash B U C 562 \backslash 12-J u 1-2$ $005 \backslash 0 \backslash \backslash \#$ B3LYP $/ 6-31+\mathrm{G}(\mathrm{D}, \mathrm{P}) \quad \mathrm{OPT}=(\mathrm{TS}, \mathrm{CALCFC}, \mathrm{NOEIGENTEST})$ MAXDISK $=20000 \mathrm{MB}$

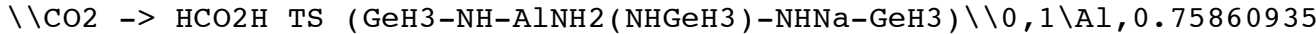
$93,0.5988658782,-0.5498415998 \backslash \mathrm{N}, 0.4756117174,0.4423964584,1.3008109806$ $\backslash \mathrm{N},-0.9371742155,1.2447223712,-1.1786705116 \backslash \mathrm{N}, 2.3750625161,1.343367140$ $1,-0.8978885281 \backslash \mathrm{N}, 0.5262670782,-1.126991009,-1.2451182173 \backslash \mathrm{Ge}, 1.8491151$ $211,0.3966741504,2.50368514 \backslash \mathrm{H}, 2.6159785538,1.732234142,2.6183463619 \backslash \mathrm{H}$, $1.2862795273,0.0532050684,3.9021966222 \backslash \mathrm{H}, 2.828136995,-0.7108738741,2.0$ $853696261 \backslash \mathrm{Ge},-1.7535694099,2.8751907208,-0.9751756975 \backslash \mathrm{H},-1.0991113896$, $3.9478939077,-1.8601496027 \backslash \mathrm{H},-3.2422703292,2.7502381028,-1.3374999992 \backslash$ $\mathrm{H},-1.6174688503,3.2664183948,0.5054392005 \backslash \mathrm{Na},-0.6876382439,-1.57007813$ $39,0.8222184897 \backslash 0,-2.8884924088,-2.1408734999,0.9604660889 \backslash \mathrm{C},-3.624615$ $5003,-1.4079518801,0.3528786432 \backslash 0,-4.6620550426,-1.0283013798,-0.06030$ $70439 \backslash \mathrm{H},-2.5101749721,-0.2572622229,-0.2712976351 \backslash \mathrm{H},-1.8883978117,0.33$ $26727093,-0.6278500399 \backslash \mathrm{Ge}, 1.6628571771,-2.5356054081,-1.3226598566 \backslash \mathrm{H}, 2$ $.7328949165,2.1746047109,-0.4420738228 \backslash \mathrm{H}, 1.4361210615,-3.4252846297,-0$ $.0626992834 \backslash \mathrm{H}, 1.3585952184,-3.4108559735,-2.5506641061 \backslash \mathrm{H}, 3.1444501476$, $-2.1237149019,-1.3121826918 \backslash \mathrm{H},-1.0244810383,0.9889851961,-2.1660371089$ $\backslash \mathrm{H},-0.1199542915,-1.1793490125,-2.0289057336 \backslash \mathrm{H},-0.2611257232,1.0464407$ $064,1.6660738328 \backslash \mathrm{H}, 2.8674375135,1.2165935088,-1.7741952416 \backslash \backslash$ Version=IA $64 \mathrm{~L}-\mathrm{G} 03$ RevC . 02 $\backslash$ State $=1-\mathrm{A} \backslash \mathrm{HF}=-7047.2155999 \backslash \mathrm{RMSD}=3.885 \mathrm{e}-09 \backslash \mathrm{RMSF}=4.738 \mathrm{e}-0$ $6 \backslash$ Dipole $=0.1648617,-0.0428677,-0.1901613 \backslash P G=C 01$ [X(C1H16Al1Ge3N4Na1O2) ] $\backslash \backslash @$ 


\section{$5 \cdot \mathrm{HCO}_{2} \mathrm{H}$}

$1 \backslash 1 \backslash G I N C-A C 3 \backslash F O p t \backslash R B 3 L Y P \backslash 6-31+G(d, p) \backslash C 1 H 16 A l 1 G e 3 N 4 N a 102 \backslash B U C 562 \backslash 12-J u l-$ $2005 \backslash 0 \backslash \backslash \#$ B3LYP $/ 6-31+G(D, P)$ OPT MAXDISK=20000MB \\HCO2H - GeH3-NH-AlNH2 $(\mathrm{NHGeH} 3)-\mathrm{NHNa}-\mathrm{GeH} 3 \backslash \backslash 0,1 \backslash \mathrm{Al}, 0.5720956071,0.5496269952,-0.6098120599 \backslash \mathrm{N}, 0$ $.4658935823,0.5691824331,1.2809957538 \backslash \mathrm{N},-1.1697347286,0.9246215233,-1$. $1780339405 \backslash \mathrm{N}, 2.1295694596,1.3902288589,-1.0629914493 \backslash \mathrm{N}, 0.5932298927,-1$ $.2702558646,-1.1083275277 \backslash \mathrm{Ge}, 1.8649413849,1.0627469906,2.3359706721 \backslash \mathrm{H}$, $2.3550917526,2.5086216566,2.0983171121 \backslash \mathrm{H}, 1.4410765381,0.9576957739,3.8$ $220146177 \backslash \mathrm{H}, 3.0347239023,0.0902030584,2.1054624085 \backslash \mathrm{Ge},-2.1609824685,2$. $4320698854,-1.0705768382 \backslash \mathrm{H},-1.7995231096,3.1537789977,0.2433113344 \backslash \mathrm{H},-$ $1.9652261965,3.4114353225,-2.2497389193 \backslash \mathrm{H},-3.6715202206,2.0877166365$,$1.0128600261 \backslash \mathrm{Na},-0.1678655185,-1.6817637322,1.1623185187 \backslash 0,-2.41858813$ $79,-2.0390765112,1.059232589 \backslash \mathrm{C},-3.0465547751,-1.2987521338,0.312190699$ $1 \backslash 0,-4.3621515521,-1.3969657351,0.1319066776 \backslash \mathrm{H},-2.5840810938,-0.486312$ $4309,-0.27929379 \backslash \mathrm{H},-4.6993011969,-2.1308371215,0.6787057315 \backslash \mathrm{Ge}, 1.88397$ $75977,-2.5264735474,-1.2216701758 \backslash \mathrm{H}, 2.3470644496,2.3410742495,-0.78482$ $98075 \backslash \mathrm{H}, 1.9391896342,-3.3011652981,0.1385426412 \backslash \mathrm{H}, 1.5439503397,-3.5731$ $346795,-2.2997928942 \backslash \mathrm{H}, 3.295625338,-1.9613711197,-1.4505587786 \backslash \mathrm{H},-1.31$ $50161938,0.5222146633,-2.1063154534 \backslash \mathrm{H},-0.1513453241,-1.4946263457,-1.7$ $643471404 \backslash \mathrm{H},-0.3833834752,1.0204817674,1.6224656199 \backslash \mathrm{H}, 2.6005229467,1.2$ $059005897,-1.9419448268 \backslash \backslash$ Version=IA64L-G03RevC.02 $\backslash$ State $=1-A \backslash H F=-7047.2$ $591351 \backslash \mathrm{RMSD}=3.240 \mathrm{e}-09 \backslash \mathrm{RMSF}=6.779 \mathrm{e}-06 \backslash \mathrm{Dipole}=-1.4886839,-1.6750465,0.51$ $05155 \backslash P G=C 01 \quad[X(C 1 \mathrm{H} 16 \mathrm{Al} 1 \mathrm{Ge} 3 \mathrm{~N} 4 \mathrm{Na} 1 \mathrm{O} 2)] \backslash \backslash @$

\section{$\operatorname{TS}\left(5, \mathrm{HCO}_{2} \mathrm{H}\right)$}

$1 \backslash 1 \backslash G I N C-A C 3 \backslash F T S \backslash R B 3 L Y P \backslash 6-31+G(d, p) \backslash C 1 H 18 A 11 \mathrm{Ge} 3 N 4 N a 102 \backslash B U C 562 \backslash 11-J u l-2$ $005 \backslash 0 \backslash \backslash \#$ B3LYP $/ 6-31+\mathrm{G}(\mathrm{D}, \mathrm{P}) \quad \mathrm{OPT}=(\mathrm{TS}, \mathrm{CALCFC}, \mathrm{NOEIGENTEST})$ MAXDISK $=20000 \mathrm{MB}$ $\backslash \backslash \mathrm{HCO} 2 \mathrm{H} \rightarrow \mathrm{CH} 2(\mathrm{OH}) 2$ TS $(\mathrm{GeH} 3-\mathrm{NH}-\mathrm{AlNH} 2(\mathrm{NHGeH} 3)-\mathrm{NHNa}-\mathrm{GeH} 3) \backslash \backslash 0,1 \backslash \mathrm{Al}, 0.708$ $7386717,0.3517567854,-0.594000387 \backslash \mathrm{N}, 0.2861172011,0.5430187225,1.222227$ $385 \backslash \mathrm{N},-0.9325012756,0.9367027187,-1.4159011019 \backslash \mathrm{N}, 2.3229662484,1.104085$ $4482,-0.950709881 \backslash \mathrm{N}, 0.7376577995,-1.4640766339,-1.0345859696 \backslash \mathrm{Ge}, 1.4011$ $875675,1.2270595371,2.481407826 \backslash \mathrm{H}, 1.8318666833,2.68545002,2.2024214621$ $\backslash \mathrm{H}, 0.6836450107,1.2030108593,3.8512147019 \backslash \mathrm{H}, 2.6675099097,0.35778362,2$. $5743692594 \backslash \mathrm{Ge},-1.9328626774,2.3472006566,-0.7642785405 \backslash \mathrm{H},-2.5054435344$ $, 1.9338141477,0.6027579549 \backslash \mathrm{H},-1.0790791795,3.6142759286,-0.5889117091 \backslash$ $\mathrm{H},-3.0921338469,2.6287926956,-1.7347301083 \backslash \mathrm{Na},-0.2784069044,-1.7847766$ $42,1.1663508744 \backslash 0,-2.4387083393,-1.7507442889,0.8254169634 \backslash \mathrm{C},-3.206197$ $1801,-1.5447538378,-0.1448916035 \backslash 0,-4.1948720562,-0.5993851131,-0.0480$ $634654 \backslash \mathrm{H},-2.3214059582,-0.9289559406,-1.3342499627 \backslash \mathrm{H},-1.7718908722,-0$. $1686379804,-1.3910113796 \backslash \mathrm{H},-3.5224611193,-2.3387284435,-0.8294592735 \backslash \mathrm{H}$ $,-4.0191715809,-0.0987686679,0.7651376791 \backslash \mathrm{Ge}, 2.12134287,-2.5704872289$, $-1.4010855962 \backslash \mathrm{H}, 2.6022155462,2.0287775113,-0.6440727356 \backslash \mathrm{H}, 3.1625529637$ $,-2.4629598622,-0.2734971562 \backslash \mathrm{H}, 1.6150887596,-4.0311560593,-1.459822689$ $2 \backslash \mathrm{H}, 2.8227234518,-2.2663376165,-2.745221511 \backslash \mathrm{H},-0.8196814138,1.06303039$ $66,-2.4241728807 \backslash \mathrm{H},-0.0920066752,-1.7428114859,-1.5608073502 \backslash \mathrm{H},-0.6667$ $007512,0.8429451612,1.4234936468 \backslash \mathrm{H}, 2.8900239318,0.8508921492,-1.751381$ 9838\\Version=IA64L-G03RevC.02 \State $=1-\mathrm{A} \backslash \mathrm{HF}=-7048.3990676 \backslash \mathrm{RMSD}=3.565 \mathrm{e}-$ $09 \backslash \mathrm{RMSF}=3.271 \mathrm{e}-06 \backslash \mathrm{Dipole}=-0.3320599,-0.301242,0.2438147 \backslash \mathrm{PG}=\mathrm{C} 01 \quad[\mathrm{X}(\mathrm{C} 1 \mathrm{H}]$ $8 \mathrm{Al} 1 \mathrm{Ge} 3 \mathrm{~N} 4 \mathrm{Na1O2})] \backslash \backslash @$ 


\section{$5 \cdot \mathrm{CH}_{2}(\mathrm{OH})_{2}$}

$1 \backslash 1 \backslash$ GINC-AC52 2 FOpt $\backslash R B 3 L Y P \backslash 6-31+G(d, p) \backslash C 1 H 18 A 11 G e 3 N 4 N a 102 \backslash B U C 562 \backslash 12-J u 1$ $-2005 \backslash 0 \backslash \backslash \#$ B3LYP/6-31+G(D,P) OPT MAXDISK=20000MB \\CH2(OH) 2 - GeH3-NH-A $1 \mathrm{NH} 2(\mathrm{NHGeH} 3)-\mathrm{NHNa}-\mathrm{GeH} 3 \backslash \backslash 0,1 \backslash \mathrm{Al},-0.607475956,-0.4180034744,-0.666113709$ $5 \backslash \mathrm{N},-0.4550871367,-0.6974489556,1.1952898215 \backslash \mathrm{N}, 1.214514475,-0.43073713$ $17,-1.1846522223 \backslash \mathrm{N},-1.9960206713,-1.4036908626,-1.309728498 \backslash \mathrm{N},-0.89644$ $97973,1.4293293312,-0.9084823912 \backslash \mathrm{Ge},-1.747765126,-1.4950152262,2.18781$ $67422 \backslash \mathrm{H},-1.9750210576,-2.9843725818,1.8372537284 \backslash \mathrm{H},-1.3565447725,-1.43$ $26473039,3.6855741857 \backslash \mathrm{H},-3.0696671259,-0.7324674874,2.0008401374 \backslash \mathrm{Ge}, 2$. $4102999776,-1.8111443475,-1.1788229156 \backslash \mathrm{H}, 2.7940235474,-2.1379574231,0$. $2790968715 \backslash \mathrm{H}, 1.8323568649,-3.0987962774,-1.7996823702 \backslash \mathrm{H}, 3.6676322345,-$ $1.4056255674,-1.9689104966 \backslash \mathrm{Na}, 0.0458012902,1.5926729341,1.3379363161 \backslash \mathrm{O}$ $, 2.1831346157,1.1465419582,0.7271123426 \backslash \mathrm{C}, 3.4586230857,1.6561955362,0$. $4328419817 \backslash 0,4.4377863637,0.6424618867,0.3367241329 \backslash \mathrm{H}, 3.477892341,2.15$ $61743743,-0.54314399 \backslash \mathrm{H}, 1.8637176612,0.5551482163,-0.063912112 \backslash \mathrm{H}, 3.7070$ $633647,2.378826051,1.2213426275 \backslash \mathrm{H}, 4.4801878579,0.1743074483,1.18286308$ $32 \backslash \mathrm{Ge},-2.3916044761,2.4419424354,-0.984119149 \backslash \mathrm{H},-2.0975231632,-2.39928$ $35158,-1.1473981245 \backslash \mathrm{H},-3.3153666338,2.0356187611,0.1796042418 \backslash \mathrm{H},-2.024$ $7639805,3.9353496548,-0.7938617178 \backslash \mathrm{H},-3.1864726678,2.3346794162,-2.303$ $814898 \backslash \mathrm{H}, 1.3131968722,0.0324942757,-2.0900245532 \backslash \mathrm{H},-0.1690387949,1.858$ $282594,-1.4793908173 \backslash \mathrm{H}, 0.4584737188,-1.0566736846,1.4744604799 \backslash \mathrm{H},-2.46$ $43494824,-1.1748362763,-2.1794378791 \backslash \backslash$ Version=IA64L-G03RevC.02 $\backslash$ State $=1$ $-\mathrm{A} \backslash \mathrm{HF}=-7048.4633016 \backslash \mathrm{RMSD}=3.576 \mathrm{e}-09 \backslash \mathrm{RMSF}=6.391 \mathrm{e}-06 \backslash \mathrm{Dipole}=0.7274849,1.4$ $917856,0.9385013 \backslash \mathrm{PG}=\mathrm{C} 01 \quad[\mathrm{X}(\mathrm{C} 1 \mathrm{H} 18 \mathrm{~A} 11 \mathrm{Ge} 3 \mathrm{~N} 4 \mathrm{Na} 1 \mathrm{O} 2)] \backslash \backslash @$

\section{$5 \cdot \mathrm{CH}_{2} \mathrm{O}$}

$1 \backslash 1 \backslash \mathrm{GINC}-\mathrm{AC} 52 \backslash \mathrm{FOpt} \backslash \mathrm{RB} 3 \mathrm{LYP} \backslash 6-31+\mathrm{G}(\mathrm{d}, \mathrm{p}) \backslash \mathrm{C} 1 \mathrm{H} 16 \mathrm{Al} 1 \mathrm{Ge} 3 \mathrm{~N} 4 \mathrm{Na} 101 \backslash \mathrm{BUC5} 62 \backslash 12-\mathrm{Ju} 1$ $-2005 \backslash 0 \backslash \backslash \#$ B $3 L Y P / 6-31+G(D, P)$ OPT MAXDISK=20000MB \\CH2O GeH3-NH-AlNH2 (N HGeH3 ) -NHNa-GeH $3 \backslash \backslash 0,1 \backslash \mathrm{Al},-0.094195201,-0.6121337702,-0.6541843423 \backslash \mathrm{N},-0$ $.0191702915,-0.6862810012,1.2355057948 \backslash \mathrm{N}, 1.6187921903,-0.0369248699,-1$ $.1438683547 \backslash \mathrm{N},-1.0316014858,-2.0671911186,-1.2364237301 \backslash \mathrm{N},-0.983674102$ $7,1.0031167981,-1.063031622 \backslash \mathrm{Ge},-1.1433999787,-1.7309292875,2.219574891$ $7 \backslash \mathrm{H},-0.9921889288,-3.2461883498,1.9597988708 \backslash \mathrm{H},-0.8737735837,-1.491497$ $6787,3.7255294439 \backslash \mathrm{H},-2.5945774074,-1.3138092486,1.9263037057 \backslash \mathrm{Ge}, 3.2252$ $518563,-0.8546477927,-1.0878899571 \backslash \mathrm{H}, 3.5801341987,-1.6815906462,-2.344$ $0917526 \backslash \mathrm{H}, 4.3609402737,0.1913847336,-0.9095636075 \backslash \mathrm{H}, 3.2635713295,-1.77$ $70593881,0.1470374515 \backslash \mathrm{Na},-0.371287797,1.6329110617,1.1850970753 \backslash 0,1.46$ $66147625,3.018643271,1.3108535704 \backslash C, 2.431844059,2.7597448992,0.6064097$ $707 \backslash \mathrm{H}, 2.4105606375,1.892506406,-0.0803824603 \backslash \mathrm{H}, 3.3354024465,3.39422798$ $6,0.650810381 \backslash \mathrm{Ge},-2.7246588121,1.4820088844,-1.1228760862 \backslash \mathrm{H},-0.7923212$ $539,-3.0203613277,-0.9862337941 \backslash \mathrm{H},-3.1175946523,2.1004665244,0.2581484$ $574 \backslash \mathrm{H},-2.9544633027,2.5853727137,-2.1732164568 \backslash \mathrm{H},-3.6882454396,0.30782$ $75433,-1.3633026146 \backslash \mathrm{H}, 1.5623452084,0.4815636893,-2.0224303411 \backslash \mathrm{H},-0.462$ $1386614,1.5636178183,-1.7331921592 \backslash \mathrm{H}, 0.9303659044,-0.7890461022,1.5939$ $913666 \backslash \mathrm{H},-1.5038981116,-2.0841692956,-2.1333228193 \backslash \backslash$ Version=IA64L-G03R evC.02 $\backslash$ State $=1-A \backslash H F=-6971.992293 \backslash \mathrm{RMSD}=9.417 \mathrm{e}-09 \backslash \mathrm{RMSF}=2.801 \mathrm{e}-06 \backslash \mathrm{Dipole}=$ $0.8175197,1.8317169,0.3249044 \backslash \mathrm{PG}=\mathrm{C} 01 \quad[\mathrm{X}(\mathrm{C} 1 \mathrm{H} 16 \mathrm{Al} 1 \mathrm{Ge} 3 \mathrm{~N} 4 \mathrm{Na1O})] \backslash \backslash \mathrm{C}$ 


\section{$\operatorname{TS}\left(5, \mathrm{CH}_{2} \mathrm{O}\right)$}

$1 \backslash 1 \backslash G I N C-A C 4 \backslash F T S \backslash R B 3 L Y P \backslash 6-31+G(d, p) \backslash C 1 H 18 A 11$ Ge3N4Na101 \BUC562\13-Jul-2 $005 \backslash 0 \backslash \backslash \#$ B3LYP $/ 6-31+G(D, P) \quad O P T=(T S, C A L C F C, N O E I G E N T E S T)$ MAXDISK $=20000 \mathrm{MB}$

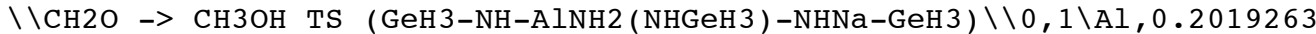
$751,-0.0145498783,-0.6847488175 \backslash \mathrm{N},-0.4667805986,0.8666907952,0.8366017$ $282 \backslash \mathrm{N},-1.3308623552,-1.0118963998,-1.2379186101 \backslash \mathrm{N}, 1.0089499538,1.20623$ $64368,-1.772188168 \backslash \mathrm{N}, 1.496721427,-1.2476500626,-0.1335365321 \backslash \mathrm{Ge},-0.240$ $5683018,2.6573516017,1.0729896257 \backslash \mathrm{H},-0.7869345439,3.5084058188,-0.0956$ $588731 \backslash \mathrm{H},-0.9788307539,3.0909747106,2.3606749341 \backslash \mathrm{H}, 1.2568702575,2.9706$ $028326,1.2396478843 \backslash \mathrm{Ge},-2.878934616,-0.0193458946,-1.4452751578 \backslash \mathrm{H},-3.5$ $950057193,0.0326855864,-0.0837976459 \backslash \mathrm{H},-2.5838249245,1.4118793395,-1.9$ $166251225 \backslash \mathrm{H},-3.7948105124,-0.7120565939,-2.4664622066 \backslash \mathrm{Na}, 0.767731723,-$ $0.7174136281,2.1164556753 \backslash 0,-0.7231506465,-2.2517788924,2.5389973067 \backslash \mathrm{C}$ $,-1.6062964236,-2.9759851518,2.0307392296 \backslash \mathrm{H},-2.6726634855,-2.778255913$ $7,2.2261057867 \backslash \mathrm{H},-1.8680879602,-2.614106875,0.444936163 \backslash \mathrm{H},-1.679044265$ $,-2.0363878149,-0.2001319709 \backslash \mathrm{H},-1.3763662044,-4.0086327083,1.719879504$ $5 \backslash \mathrm{Ge}, 3.2788952959,-1.0846484022,-0.4634203025 \backslash \mathrm{H}, 0.5914213719,2.0911378$ $026,-2.0365210427 \backslash \mathrm{H}, 3.814730083,0.1712113885,0.2473710574 \backslash \mathrm{H}, 4.01591599$ $66,-2.3179075931,0.1054331166 \backslash \mathrm{H}, 3.6274013999,-0.9803996882,-1.96606083$ $02 \backslash \mathrm{H},-1.1823154558,-1.5944703228,-2.0627200803 \backslash \mathrm{H}, 1.2118644187,-2.22529$ $6092,-0.1803660643 \backslash \mathrm{H},-1.3915200829,0.5881258485,1.1613742319 \backslash \mathrm{H}, 1.68733$ $71757,0.9522315047,-2.4808926923 \backslash \backslash$ Version=IA64L-G03RevC.02 $\backslash$ State $=1-A \backslash H$ $\mathrm{F}=-6973.149868 \backslash \mathrm{RMSD}=5.187 \mathrm{e}-09 \backslash \mathrm{RMSF}=4.203 \mathrm{e}-06 \backslash \mathrm{Dipole}=-0.2386478,-0.8002$ $988,0.5428293 \backslash \mathrm{PG}=\mathrm{C} 01 \quad[\mathrm{X}(\mathrm{C} 1 \mathrm{H} 18 \mathrm{~A} 11 \mathrm{Ge} 3 \mathrm{~N} 4 \mathrm{Na} 1 \mathrm{O} 1)] \backslash \backslash @$

\section{$5 \cdot \mathrm{CH}_{3} \mathrm{OH}$}

$1 \backslash 1 \backslash$ GINC-AC9 $\backslash$ FOpt $\backslash R B 3 L Y P \backslash 6-31+G(d, p) \backslash C 1 H 18 A l 1 G e 3 N 4 N a 101 \backslash B U C 562 \backslash 12-J u l-$ $2005 \backslash 0 \backslash \backslash \#$ B $3 L Y P / 6-31+G(D, P) \quad O P T$ MAXDISK $=20000 \mathrm{MB} \backslash \backslash \mathrm{CH} 3 \mathrm{OH}$ GeH3-NH-AlNH2 (N $\mathrm{HGeH} 3)-\mathrm{NHNa}-\mathrm{GeH} 3 \backslash \backslash 0,1 \backslash \mathrm{Al},-0.2953647715,-0.3909639298,-0.6721778676 \backslash \mathrm{N},-$ $0.2510910031,-0.7706625389,1.1771000556 \backslash \mathrm{N}, 1.546075436,-0.1955625465,-1$ $.0610376554 \backslash \mathrm{N},-1.513816008,-1.4752541767,-1.481807559 \backslash \mathrm{N},-0.7709115707$, $1.4268964687,-0.8279080312 \backslash \mathrm{Ge},-1.4886402317,-1.7891524016,2.0281658608$ $\backslash \mathrm{H},-1.5044241322,-3.2655201349,1.564424046 \backslash \mathrm{H},-1.1983524732,-1.79144837$ $78,3.5496690804 \backslash \mathrm{H},-2.8847918379,-1.1846299591,1.8033764423 \backslash \mathrm{Ge}, 2.846353$ $0773,-1.4652696872,-1.1008979778 \backslash \mathrm{H}, 2.9635859572,-2.161565626,-2.472790$ $0492 \backslash \mathrm{H}, 4.2226060871,-0.8406037691,-0.7710039775 \backslash \mathrm{H}, 2.5287500923,-2.5159$ $785905,-0.022214294 \backslash \mathrm{Na},-0.042133177,1.5434966307,1.5005359534 \backslash 0,2.1558$ $482674,1.4559908288,1.0123070695 \backslash \mathrm{C}, 3.251426684,2.3298490315,0.77100487$ $68 \backslash \mathrm{H}, 4.1976774958,1.7771340431,0.7112160076 \backslash \mathrm{H}, 3.1203546151,2.902072604$ $9,-0.1583292726 \backslash \mathrm{H}, 2.0194841755,0.8443006095,0.2121307867 \backslash \mathrm{H}, 3.319035756$ $4,3.0355072068,1.6041904679 \backslash \mathrm{Ge},-2.373974027,2.2439924766,-1.0097726283$ $\backslash \mathrm{H},-1.5516378852,-2.4795905888,-1.3504109522 \backslash \mathrm{H},-3.3521021471,1.6443248$ $394,0.0173902155 \backslash \mathrm{H},-2.2316978359,3.7556230381,-0.6983612673 \backslash \mathrm{H},-3.00626$ $49434,2.129014632,-2.4148489784 \backslash \mathrm{H}, 1.6591585536,0.3869283145,-1.8933602$ $002 \backslash \mathrm{H},-0.0530695664,1.9779928874,-1.2968365973 \backslash \mathrm{H}, 0.6776176884,-1.04506$ $61008,1.4994963089 \backslash \mathrm{H},-1.9295090424,-1.2556205525,-2.3800826117 \backslash \backslash$ Versio $\mathrm{n}=\mathrm{IA} 64 \mathrm{~L}-\mathrm{G} 03 \mathrm{RevC} .02 \backslash$ State $=1-\mathrm{A} \backslash \mathrm{HF}=-6973.2298653 \backslash \mathrm{RMSD}=9.227 \mathrm{e}-09 \backslash \mathrm{RMSF}=4.90$ $1 \mathrm{e}-05 \backslash \mathrm{Dipole}=0.8692559,1.6248665,0.6801671 \backslash \mathrm{PG}=\mathrm{C} 01 \quad[\mathrm{X}(\mathrm{C} 1 \mathrm{H} 18 \mathrm{Al} 1 \mathrm{Ge} 3 \mathrm{~N} 4 \mathrm{Na} 1 \mathrm{O}$ $1)] \backslash \backslash @$ 
Table S2. MP2/G3MP2Large Total Energies (0 K, hartrees)

\begin{tabular}{|c|c|c|c|c|c|}
\hline & $\mathrm{H}_{2}$ & -1.152796576 & & & \\
\hline & $\mathrm{CO}_{2}$ & -188.2899394 & & & \\
\hline & $\mathrm{HCO}_{2} \mathrm{H}$ & -189.4273567 & & & \\
\hline & $\mathrm{CH}_{2} \mathrm{OH}_{2}$ & -190.5807061 & & & \\
\hline & $\mathrm{H}_{2} \mathrm{O}$ & -76.29391303 & & & \\
\hline & $\mathrm{CH}_{2} \mathrm{O}$ & -114.2740342 & & & \\
\hline & $\mathrm{CH}_{3} \mathrm{OH}$ & -115.4577208 & & & \\
\hline & Catalyst & Substrate & $\mathrm{Cat} \bullet \mathrm{S}$ & TS & $\mathrm{Cat} \bullet \mathrm{P}$ \\
\hline \multirow[t]{3}{*}{1} & -1416.384527 & $\mathrm{CO}_{2}$ & -1604.68454 & -1605.770916 & -1605.828927 \\
\hline & & $\mathrm{HCO}_{2} \mathrm{H}$ & -1605.828927 & -1606.924039 & -1606.989113 \\
\hline & & $\mathrm{CH}_{2} \mathrm{O}$ & -1530.679196 & -1531.788373 & -1531.86898 \\
\hline \multirow[t]{3}{*}{2} & -1577.903967 & $\mathrm{CO}_{2}$ & -1766.20521 & -1767.303996 & -1767.359878 \\
\hline & & $\mathrm{HCO}_{2} \mathrm{H}$ & -1767.359878 & -1768.443032 & -1768.516138 \\
\hline & & $\mathrm{CH}_{2} \mathrm{O}$ & -1692.19829 & -1693.305832 & -1693.395443 \\
\hline \multirow[t]{3}{*}{3} & -1336.754312 & $\mathrm{CO}_{2}$ & -1525.052889 & -1526.144116 & -1526.194581 \\
\hline & & $\mathrm{HCO}_{2} \mathrm{H}$ & -1526.194581 & -1527.283455 & -1527.352578 \\
\hline & & $\mathrm{CH}_{2} \mathrm{O}$ & -1451.039346 & -1452.144471 & -1452.230442 \\
\hline \multirow[t]{3}{*}{4} & -6776.295102 & $\mathrm{CO}_{2}$ & -6964.595979 & -6965.689649 & -6965.742908 \\
\hline & & $\mathrm{HCO}_{2} \mathrm{H}$ & -6965.742908 & -6966.835307 & -6966.900953 \\
\hline & & $\mathrm{CH}_{2} \mathrm{O}$ & -6890.586287 & -6891.698512 & -6891.779668 \\
\hline \multirow[t]{3}{*}{5} & -6858.164852 & $\mathrm{CO}_{2}$ & -7046.4684 & -7047.582039 & -7047.616828 \\
\hline & & $\mathrm{HCO}_{2} \mathrm{H}$ & -7047.616828 & -7048.723812 & -7048.779367 \\
\hline & & $\mathrm{CH}_{2} \mathrm{O}$ & -6972.460729 & -6973.58522 & -6973.65831 \\
\hline
\end{tabular}


Table S3. Full Citations for References 1 and 9.

1. Hoffert, M. I.; Caldeira, K.; Benford, G.; Criswell, D. R.; Green, C.; Herzog, H.; Jain, A. K.; Kheshgi, H. S.; Lackner, K. S.; Lewis, J. S.; Lightfoot, H. D.; Manheimer, W.; Mankins, J. C.; Mauel, M. E.; Perkins, L. J.; Schlesinger, M. E.; Volk, T.; Wigley, T. M. L. Science 2002, 298, 981.

9. Gaussian 03, Revision B.03, M. J. Frisch, G. W. Trucks, H. B. Schlegel, G. E. Scuseria, M. A. Robb, J. R. Cheeseman, J. A. Montgomery, Jr., T. Vreven, K. N. Kudin, J. C. Burant, J. M. Millam, S. S. Iyengar, J. Tomasi, V. Barone, B. Mennucci, M. Cossi, G. Scalmani, N. Rega, G. A. Petersson, H. Nakatsuji, M. Hada, M. Ehara, K. Toyota, R. Fukuda, J. Hasegawa, M. Ishida, T. Nakajima, Y. Honda, O. Kitao, H. Nakai, M. Klene, X. Li, J. E. Knox, H. P. Hratchian, J. B. Cross, C. Adamo, J. Jaramillo, R. Gomperts, R. E. Stratmann, O. Yazyev, A. J. Austin, R. Cammi, C. Pomelli, J. W. Ochterski, P. Y. Ayala, K. Morokuma, G. A. Voth, P. Salvador, J. J. Dannenberg, V. G. Zakrzewski, S. Dapprich, A. D. Daniels, M. C. Strain, O. Farkas, D. K. Malick, A. D. Rabuck, K. Raghavachari, J. B. Foresman, J. V. Ortiz, Q. Cui, A. G. Baboul, S. Clifford, J. Cioslowski, B. B. Stefanov, G. Liu, A. Liashenko, P. Piskorz, I. Komaromi, R. L. Martin, D. J. Fox, T. Keith, M. A. Al-Laham, C. Y. Peng, A. Nanayakkara, M. Challacombe, P. M. W. Gill, B. Johnson, W. Chen, M. W. Wong, C. Gonzalez, and J. A. Pople, Gaussian, Inc., Pittsburgh PA, 2003. 